\title{
Robust, Resilient and Reliable Architecture for V2X Communication
}

\author{
Muhammad Awais Khan, Saptarshi Ghosh, Sherif Adeshina Busari, Kazi Mohammed Saidul Huq, Tasos \\ Dagiuklas, Shahid Mumtaz, Muddesar Iqbal and Jonathan Rodriguez
}

\begin{abstract}
The new developments in mobile edge computing (MEC) and vehicle-to-everything (V2X) communications has positioned 5G and beyond in a strong position to answer the market need towards future emerging intelligent transportation systems and smart city applications. The major attractive features of $\mathrm{V} 2 \mathrm{X}$ communication is the inherent ability to adapt to any type of network, device, or data, and to ensure robustness, resilience and reliability of the network, which is challenging to realize. In this work, we propose to drive these further these features by proposing a novel robust, resilient and reliable architecture for V2X communication based on harnessing MEC and blockchain technology. A three stage computing service is proposed. Firstly, a hierarchcial computing architecture is deployed spanning over the vehicular network that constitutes cloud computing (CC), edge computing (EC), fog computing (FC) nodes. The resources and data bases can migrate from the high capacity cloud services (furthest away from the individual node of the network) to the edge (medium) and low level fog node, according to computing service requirements. Secondly, the resource allocation filters the data according to its significance, and rank the nodes according to their usability, and selects the network technology according to their physical channel characteristics. Thirdly, we propose a blockchain-based transaction service that ensures reliability. We discussed two use cases for experimental analysis, plugin electric vehicles in smart grid scenarios, and massive IoT data services for autonomous cars. The results show that car connectivity prediction is accurate $98 \%$ of the times, where $92 \%$ more data blocks are added using micro-blockchain solution compared to the public blockchain, where it is able to reduce the time to sign and compute the proof-of-work (PoW), and deliver a low-overhead Proof-of-Stake (PoS) consensus mechanism. This approach can be considered a strong candidate architecture for future V2X, and with more general application for everythingto-everything (X2X) communications.
\end{abstract}

Index Terms-Architecture, Cyber-physical systems (CPS), Vehicle-to-everything (V2X), Robust, Resilient, Reliable, Smart Grids, Blockchain, vehicular sensor networks (VSNs), 5G.

This research was supported by Instituto de Telecomunicações - Aveiro and funded by National Funds through FCT - Fundação para a Ciência e a Tecnologia (FCT-Portugal). The research leading to these results received funding from the European Commission H2020 program under grant agreements no. 815178 (5GENESIS project) and MSCA-IF-2018-839573 (5GACE project). (Corresponding author: Muhammad Awais Khan)

M. A. Khan, S. A. Busari, S. Mumtaz and J. Rodriguez are with the Instituto de Telecomunicações, Aveiro, Portugal (e-mail: \{jadoon.awais, sherifbusari, smumtaz, jonathan\}@av.it.pt).

K. M. S. Huq and J. Rodriguez are with the Faculty of Computing, Engineering and Science, University of South Wales, Pontypridd, United Kingdom (e-mail: \{kazi.huq, jonathan.rodriguez\}@southwales.ac.uk).

S. Ghosh, T. Dagiuklas and M. Iqbal are with the Department of Computer Science and Informatics, School of Engineering, London South Bank University, United Kingdom (e-mail: $\{$ ghoshs4, tdagiuklas, m.iqbal $\} @ 1$ lsbu.ac.uk).

\section{INTRODUCTION}

Cyber-physical systems (CPS) are turning everything around us into digital data products and services. CPS are used to connect all devices through gateways to the central cloud platform requiring a robust, resilient and reliable architectures [1]. Vehicular networks are characterized by high mobility and this leads to latency, reliability and availability challenges. Next generation vehicular networks require architectures that are capable of operating in diverse environments with respect to deployment topology, network type, security and spectral bands. For example, vehicle-to-everything (V2X) communication requires large bandwidth and computational resources to support the large array of sensors and communication chipsets for the semi- and fully-autonomous vehicles with respect to the required real applications such as traffic monitoring, fleet management, distant support for safety and diagnostics [2][5].

In V2X, the in-built vehicle sensor platforms use centralization of different functionalities through a built-in server of the vehicle connected via many actuators, such as CAN, $\mathrm{Wi}-\mathrm{Fi}$, and Bluetooth technologies. The complexity of these centralized implementation of in-built sensors to the outside world is dependent on how much services are required from outside resources. With the emerging setups like vehicleto-grid (V2G) [6]-[8], vehicle-to-pedestrian (V2P), vehicle to industrial internet of things (V2IoT), many features are needed to connect the in-vehicle sensors and the outside world for service flexibility. However, the existing supporting networking functions [9], protocols, consumer technologies and architectures are not efficient.

Different standardizations have already addressed the safety-critical applications of the V2X by using redundant and multiple communication channels and low delay technologies [10]-[12]. The available solutions have cost and timing challenges to address as well [13]-[16]. Currently, V2X rely on multi-technology and multi-network capabilities in disseminating and collecting information among the vehicles [17]-[20]. Technical enhancements (network orchestration, edge computing enhancements, end-to-end security and multiconnectivity operation) are also big issues. Moreover, the provision of acceptable levels of services in case of faults, errors or targeted attacks are big challenges. Therefore, more than ever before, the new trends and technical enhancements in V2X communications need a holistic, robust, resilient and reliable architecture to tackle the current issues [21]-[26].

In a robust vehicular network, the overall system must have 
the ability to cope with the loss of individual nodes and elements of the network. In communication networks, network robustness is mostly promoted by redundancy and absence of leader in the network. The new V2X paradigms may need scalability to perform well with different sizes and structures of the network. The introduction or removal of nodes must not result in drastic change in the overall performance of the network. To perform many tasks in many different environments, the architecture must be resilient yet flexible with broad spectrum and many different technologies available today. In this paper, a reliable, robust and resilient architecture for $\mathrm{V} 2 \mathrm{X}$ communication is presented. The next subsection provides the current design and evaluation of available architectures, discussing their strengths and weaknesses.

\section{A. Related Work}

The principle of a vehicular system is related to the swarm ecosystem. Each node moves independently and has sensing and communication capabilities. The individual node may or may not have access to centralized global knowledge and may consent to cooperate with neighboring nodes to tackle any given task [27]. In [28], a survey on hybrid architectures for V2X communication using dedicated short-range communications (DSRC) and cellular networks is presented. A largescale urban road network model is designed for data collection and dissemination in [29]. The model collects, diffuse and disseminate the information. The congestion information and automatic update generation are communicated to the nodes.

The geographical information of the vehicle is taken from the global positioning system (GPS) and geographic information system (GIS) giving travel time and distance covered by the vehicle. The information is fused to provide compact, but precise information by checking the congestion, and in case of slow mobility, the redundant data is removed from the database. Later, the information is released to the cars using the broadcast information dissemination procedure. The update of the information set of the road are analyzed. The influence of inflow volume on dissemination, effect of congestion information, the number of vehicles on dissemination of congestion information, coverage time and coverage rate of information are calculated. In [30], a citywide traffic flow is predicted using multiple spatio-temporal convolutional neural networks (MGSTC). Multiple 3D volumes with spatial and temporal information trained the model using MGSTC and predicted the inflows and the outflows of the traffic.

Several works came up with various architectures for V2V, V2I, and I2V communications. Some of these introduced cellular technology for communication while some architectures followed combinatorial mechanisms. The best combination of the these technologies were Wi-Fi, 802.11p and cellular so far as mentioned in [31]. The framework for high performance vehicular streams for cooperative adaptive cruise control is presented using transportation and communication layered architecture. Petri net model for subplatoons is used to provide fault tolerance using GPS, WiFi, DSRC and cellular technologies. This research considers communication and stability challenges concerning communications. However, the architecture does not consider unplanned human and environmental factors, and the implementation was focused only to urban areas and to applications where small coverage areas were sufficient. The Proposed architecture considers multiple 5G technologies and the provision of slicing mechanism for EC, FC and CC. In [32], deep learning (DL) algorithms are used for distributed intelligent video surveillance (DIVS) system. Edge computing migrated the data for balanced workload and computational power at edge node using parallel training, model synchronization and workload balancing. In [33], cloud computing algorithms are proposed to improve virtual machine utilization and scheduling performance.

Geographical migration of processing using fog-enabled vehicles is proposed in [34] for smart cities. The architecture takes advantage of internet of vehicles to migrate the task from servers to fog nodes. The selection of vehicle path is calculated by resource pricing for migration model and results showed efficiency of the proposed scheme. In [35], a cellular infrastructure-to-everything application scenario is investigated with three different subarray structures for massive MIMO, such as fully-connected, sub-connected and overlapped subarray structures for the comparative analysis of spectral efficiency, energy efficiency, cost and hardware complexity. In [36], a scalable IoT datacenter using spine-leaf topology provided the enhanced fog computing. Smart IoT applications running on clouds are connected to the fog and IoT nodes with multilayer virtualization, with infrastructure monitoring to provide QoS monitoring, performance prediction, data analytics, data mining and cyber security. In [37], an architecture to disseminate the real time services using TCP/IP-based single hop connection for vehicular clients, such as, car parking system. Fog nodes provide bandwidth, computation, storage and application services to the connected vehicle clients. Hardware prototype consist of multiple sensors connected to microcontroller and GSM modules.

In [38], each functional area using deep analysis and modeling of dataset of floating cars and official data is proposed. The origin-destination (OD) matrix of social vehicles with a gravity model is predicted, and then the OD matrix is calibrated with the average growth factor method. Cooperative automated driving architecture is presented for reducing energy consumption and emissions, and improving the road safety in [39]. The architecture is platform-independent and include V2X communication, localization, state estimation, and longitudinal and lateral control. The architecture uses data age for predictive control in trajectory tracking. However, the solution is tested only for two different concept cars for automated and connected mobility.

Vehicle mobility is exploited to provide the edge assisted V2X communication in [40]. The proposed solution adopts a hybrid architecture of convolutional and recurrent neural networks for customized mobility prediction. The simulated results from the testbed deployed in Tokyo, Japan validated the proposed solution when compared with traditional methods. Adaptive cruise control systems is optimized with vehicle's longitudinal dynamics using predicted average driving profile [41]. The architecture uses cost function and needs to ensure the real time connectivity to different data sources. However, the system behavior is tuned using parameter of relative fuel 
consumption reduction. Dynamic services provisioning using smart hierarchical fog network orchestration are proposed in [42]. Fog optimizer used cost function and other constraints to predictive control strategy with respect to reference trajectory. Neural network simulation testbed simulated the SDN optimization engine that provided the feedback to the neural network predictive switch controller with a reference fog node synchronizer. In [43], multiple fixed-cycle traffic light problems for compound Poisson arrivals provide generalization for multiple lane, random departure times, left turns, and arrival rate to be a function of time.

The provision of network security, scalability and reliability is addressed for different architectures, but research is limited with other parameters. The need of blockchain is critically analyzed in [44], comparing permissionless (e.g., Bitcoin/Ethereum) and permissioned (e.g., Hyperledger/Corda) and central managed databases. In [45], a privacy-aware secure framework for $\mathrm{V} 2 \mathrm{X}$ communication is proposed using blockchain and named data networking. Vehicle plate information is used for joining the cluster using a pair of encryption keys and later verify the information. However, the research does not include the features of multi-technologies and microblockchain. In [46], a secure LTE-based V2X communication architecture is proposed. The proposed mechanism focuses on message delivery and privacy preserving security requirements in LTE systems. The robustness of the system is maintained using key distribution mechanisms in which an attacker cannot predict the international mobile subscriber identity (IMSI) from listening to the $\mathrm{V} 2 \mathrm{X}$ service registration message exchange since it is encrypted by a short term symmetric key. However, the research has an analytical framework to investigate the secure mechanism by providing the certificates and encryption mechanisms. In proposed research, we have exploited the blockchain mechanism to provide the security to any financial transaction in the whole network. The mechanism could add transaction value to the block even on the edge.

The mobility of vehicles is inherently a continuous function, but many architectures use discrete historic data for ranking data, node and network depending upon the issues of storage and processing limitation of the nodes. Therefore, several analytical models and architectures rank the data and nodes using the transmission range and node connectivity. These works provide an insight to the possible dimensions of the V2X communications. So far, to the best of the authors' knowledge, there is no general architecture available that tackles not only data and node, but also the network at the same time. Thus, proposed architecture employs deep learning based ranking mechanism and considers all three facets (i.e., data, nodes and networks) at the same time.

\section{B. Paper Contribution}

In this paper, we provide a three-fold contribution by providing a robust, resilient and reliable architecture for $\mathrm{V} 2 \mathrm{X}$ communication.

1) For V2X communication, we propose a multi-stage computational architecture in which multiple wireless access technologies are used. A close to user and very low latency computational mechanism is provided using EC. A middle layer of FC provides a low-latency network connection. FC helps in operations of compute, storage and networking between edge device and cloud resources. For high bandwidth connections, CC is provided.

2) We introduce multiple access communication technologies covering Wi-Fi, 802.11p, cellular, controller area network (CAN), LoRa, and Bluetooth. The architecture supports multiple streams of data and is designed to prioritize the data, content type, and network. The data transferred are ranked among the in-built sensors, outboard sensors, fog nodes/servers, and the outside remote world servers depending upon the criticality of the data. Machine learning algorithms are used to rank the data, nodes and network for choosing the best ones for forwarding decisions.

3) The architecture incorporates security and dependability of the network using the concept of blockchain. The distributed micro and macro level blockchain solution ensures the security by using decentralized SDN controllers with transparent rules in the network. Blockchain helps to maintain the integrity of flow rules and encryption schemes help the confidentiality of the data.

\section{Paper Organization}

The paper is organized into following sections. Section II provides the system model and architectural components. Section III explores multi-stage computing architecture using 5G solutions. The full stack architecture for EC, FC and CC is explained and the analysis of each stage is given. Section IV provides the ranking mechanisms for data, nodes, and networks for communication. The Blockchain architecture is explained in $\mathrm{V}$ and how the electronic transactions are settled using the blockchain management platform. Practical use cases of smart grid for plug-in electric vehicles and massive IoT data services in autonomous cars are outlined in Section VI. Section VII provides simulation setup, the cross platform integration and SDN implementation details. The organization of various layers and APIs' details of deep learning engine is provided as well. Section VIII provides the results and the analysis of the network connectivity, its prediction. Moreover, the simulation

$$
\begin{array}{r}
C_{A}\left(c_{i}, c_{j}\right)=\left\{c_{a_{i, j}} \in[0,1]\left|c_{a_{i, j}}=Z\left(\frac{f\left(c e\left(c_{i}\right) \times c e\left(c_{j}\right)\right)}{d\left(c_{i}, c_{j}\right)^{2}}\right)\right|\right\} \\
\text { subject to: } f\left(c e_{i}, c e_{j}\right)= \begin{cases}\operatorname{rank}\left(c t_{i}\right), & \text { if } \quad c t_{i}=c t_{j}, \quad \forall c_{i, j} \in\{c(t)\} \\
0, & \text { otherwise }\end{cases}
\end{array}
$$




$$
\left[C_{A}(t)\right]_{n \times n}=\left[\left\{c_{a_{i, j}}^{(t)}\right\}\right]_{n \times n} \sim\left(D_{\text {enter }}\left|D_{\text {depart }}\right| D_{\text {dist }}\right), \quad \forall\left\{c_{i}, c_{j}\right\} \in \mathcal{U}
$$

results of proposed micro-blockchain are compared with the public blockchain mechanism. Finally, Section IX concludes this work.

\section{SySTEM MOdEL}

Let $\mathcal{U}$ be the universe of discourse containing cars being monitored by the implemented sensor network. We assume the cars are arriving and departing $\mathcal{U}$ following a Poisson distribution [47], [48]. Furthermore, the dynamics of $\mathcal{U}$ is monitored by periodic updates at a fixed interval $\Delta t$, over a varying window size $w_{t}$ where $t$ is time. Therefore, given a time window $w_{t}=k \Delta t \mid k \in \mathbb{N}$ and a universe which is a collection of cars $\mathcal{U}=\mathcal{C}(t)=\left\{c_{i}\right\}$ such that the indexing variable $i$ is a unique identifier for each car, regardless of whether or not the car belongs in $\mathcal{U}$. Thus, $\{\mathcal{C}(t)\}$ models a stochastic process from a joint distribution of two Poisson processes (i.e., arrival and departure). Each car $c_{i} \in\{\mathcal{C}(t)\}$ is fitted with communication equipment (CE) with $c e\left(c_{i}\right)=$ $\left\{c t_{i}\right\} \subseteq P(\mathcal{C T})$, where $\mathcal{C} \mathcal{T}=c t_{i}$ is a finite, non-empty set of available technologies with their respective specifications and ranks, where $\operatorname{rank}\left(c t_{i}\right) \in[0,1]$. Each car may be equipped with several CEs of heterogeneous communication technologies $(\mathrm{CT})$. Therefore, $\left\{c t_{i}\right\}$ can be any possible subset of $\mathcal{C} \mathcal{T}$. The affinity model and system architecture that characterize the system model are described as follows.

\section{A. Affinity Model}

Let $\mathcal{C}_{A}\left(c t_{i}, c t_{j}, d\left(c_{i}, c_{j}\right)\right)$ be a real function with bounded range of $[0,1]$ and an affinity cost between two cars $c_{i}$ and $c_{j}$ separated by a distance $d\left(c_{i}, c_{j}\right)$. Since each car has multiple CEs installed, there exists several affinity costs for each CT. However, the two cars have affinity only if they share at least one common CT (since, for instance, an LTE module cannot communicate with highway addressable remote transducer protocol (HART) module). A compact form of the affinity cost function is given by (1). In wireless communications, the intensity of radio waves over distance obeys the inversesquare law in free space. In a high mobile non free space vehicular network, the signal can be reflected, scattered or absorbed by objects in the propagation environment, for which probabilistic, deterministic models are available [49]-[51].

The affinity cost function $\mathcal{C}_{A}\left(c_{i}, c_{j}\right)$ returns a set of costs $\left\{c_{a_{i, j}}\right\}$ bounded by $[0,1]$ [where $\left.\left|\left\{c_{a_{i, j}}\right\}\right|=\left|\left\{c t_{i}\right\} \cap\left\{c t_{j}\right\}\right|\right]$ corresponding to each data link. $\left[\mathcal{C}_{A}(t)\right]_{n \times n}$ given by (2) is thus a random, symmetric affinity matrix representing a stochastic process drawn from a joint distribution of entering cars $D_{\text {enter }}$, departing cars $D_{\text {depart }}$ and their varying distances $D_{\text {dist }}$ due to their respective mobility across $\mathcal{U}$.

As discussed, $D_{\text {enter }}$ and $D_{\text {depart }}$ are assumed to be the Poisson distribution [52]. However, $D_{\text {dist }}$ comes from a joint distribution of latitudinal and longitudinal mobility of the cars. In the worst case scenario, they perhaps converge to Brownian motion. Consequently, the time series converges to be nonstationary thus any predictive analysis would be infeasible. Therefore, we propose to predict the time-series analysis of the distance for each pair of cars which in turn simplifies the problem by:

(i) reducing a vector regression for two axes into an univariate time-series analysis problem;

(ii) easing the proximity calculation by expressing it as distance;

(iii) considering $D_{d i s t}$ to be a Brownian motion, thereby trivializing the proof by using central limit theorem such that the piecewise distribution of it converges to a Gaussian distribution. Hence, a recurrent neural network (RNN) with long-short term memory (LSTM) becomes an ideal choice to approximate the hidden distribution; and

(iv) representing the predicted distances of car pairs $\left\{c_{i}, c_{j}\right\}$ as $d_{i, j}^{\left(w_{h}, w_{f}\right)}$ based on the historical time window $w_{h}$ forecasted over $w_{f}$ such that the optimality is expressed as (3). This returns the optimal pair of cars having the highest probability to maintain proximity over a period of time.

$$
\arg \min _{i, j}\left(\operatorname{median}\left(d_{i, j}^{\left(w_{h}, w_{f}\right)}\right)\right)
$$

\section{B. System Architecture}

The proposed architecture has three major components as shown by Fig. 1. The first component represents the 5G computing unit with three levels of computing power (i.e, cloud computing (CC), fog computing (FC) and edge computing (EC)). The second component employs machine learning to deal with the prioritization of data, node and network. The last component features a block chain implementation for electronic transaction. These three components are described in the following.

1) 5G, SDN and Edge/Fog/Cloud Computing: 5G and beyond network architectures need to meet stringent conditions with respect to enhanced connectivity and scalability with minimal lag times in order to provide services that meet the needs of the applications. To provide different layers and interfaces for computing power depending on the peak data rates, latency, spectral efficiency, mobility and connection density, we propose three layers of computing in the proposed architecture. These layers include EC which is the closest to the users, the FC that bridges the gap between the centralized server and edge nodes, and $\mathrm{CC}$ that has the highest computational power but are typically far from the users. Virtualized logical networks provide edge computing services with least time delays, FC provides connection density and mobility with a medium latency, and $\mathrm{CC}$ for high bandwidth at the centralized server. Software defined networking (SDN) helps organize the control and data planes in creating interoperable applications 


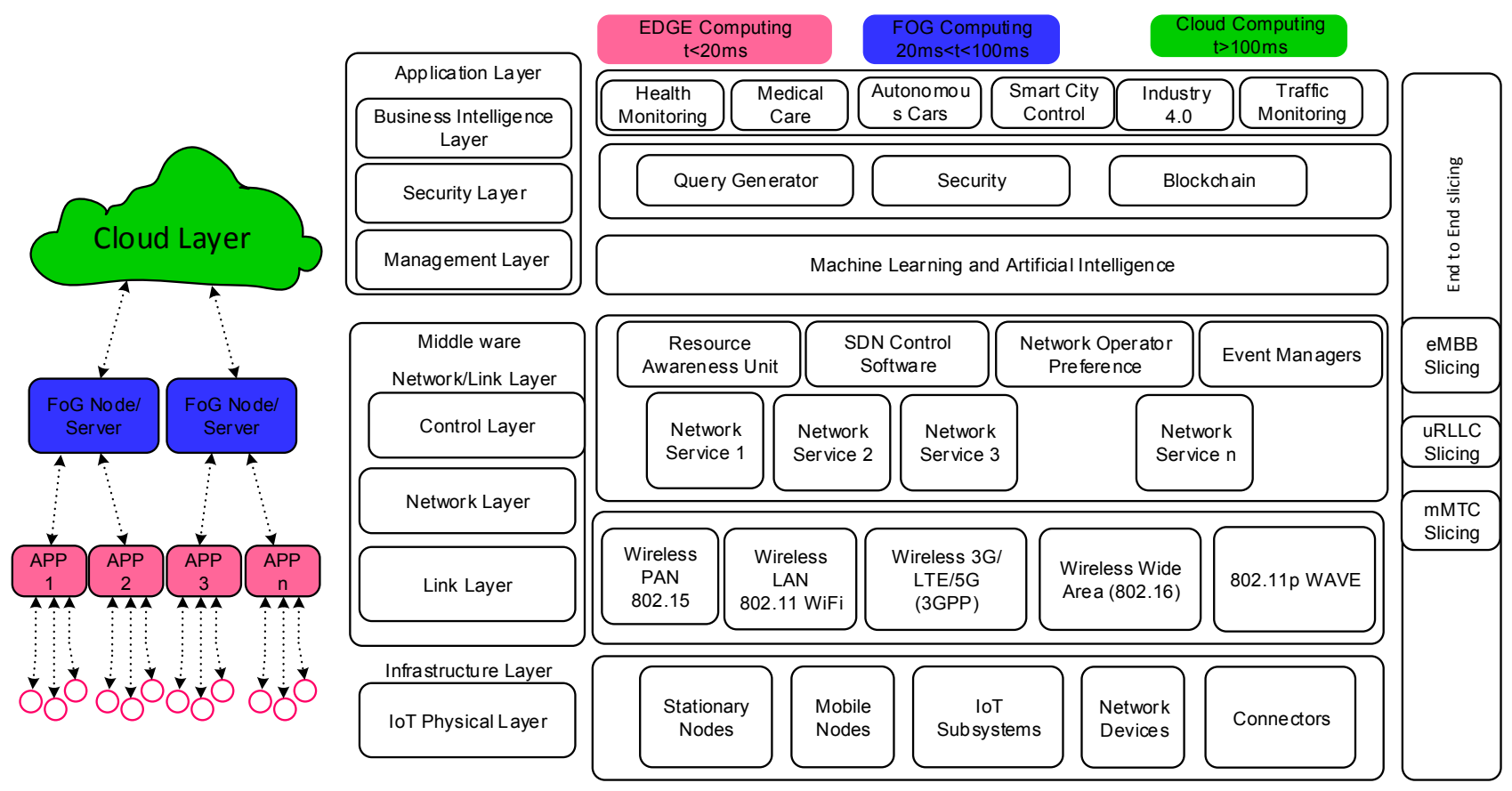

Figure 1. Proposed V2X data communication architecture

for the vehicular network ecosystem. The immense increase in the components and interfaces is to facilitate a secure, robust and resilient architecture. The resulting challenges brought about by the increase are explained in Section III.

2) Prioritization of data, nodes, and network: For a network with a massive number of vehicular nodes and sensors, a huge amount of data is generated. In the architecture, we propose cleansing and enrichment of the data before content storage or transmission, and prioritization of data according to the criticality of the data and the applications. Important nodes that are critical for the network services are also ranked depending on the data quality and assurance to work efficiently and effectively in the network. Since there are multiple network accesses provided to any node, the priority of the network is calculated based on the cost function. Further details on the ranking of the data, nodes and network are provided in Section IV.

3) Blockchain for Electronic Transactions: To enable fast and secure electronic transactions in vehicular networks, we have propose to use blockchain. As soon as a vehicle enters the network, distributed certification authority assesses and validates the node information. Then any transaction done by the vehicle is updated through the help of a distributed ledger of the blockchain. The detailed working of the blockchain will be explained in Section V.

\section{5G, SDN AND COMPUTING}

How could 5G help provide the computational power depending upon the needs of the users? Three different usages of the network are taken from 5G cellular communications. New services and markets need an architecture that could give solutions for critical communications with ultra-low latency, ultra-high reliability, and ubiquitous availability, such as industrial and tactile internet. For massive IoT, the scalability of sensor nodes and wearable devices can induce new markets, such as smart utilities, e-Health, smart homes, etc. The other important features of 5G would be the network mobility, creation of flexible functions and capabilities to provide higher data rates and coverage with the optimal resource utilization. The mathematical modeling is explained thus:

\section{A. Mathematical modeling of Edge/Fog/Cloud Computing}

The model of the V2X communication considers three levels of caching and processing power in the system $(\mathrm{CC}$, $\mathrm{FC}$ and $\mathrm{EC}$ ), such that $\mathrm{CC}>\mathrm{FC}>\mathrm{EC}$. The EC contains the processing on the computational devices, such as group of cars or even a single car with multiple processing units. The total computational power (TCP) is the sum of all the computational power of the network besides the computational resources needed to migrate the processing from each unit. If migration of processing between EC and FC is $\delta x$ and migration between FC and CC is $\delta y$, then the TCP can be written as:

$$
\begin{aligned}
T C P= & \sum(C C+F C+E C) \\
& -\sum(\delta x+\delta y)
\end{aligned}
$$

where $\operatorname{TCP}(N)=\left\{N_{t} \mid t\right\}$ belongs $[0, n-1]$ is a finite nonempty reference string of nodes at any time instant $t$ in the network. The FC is the section of the network with multiple EC units inside it. The measure of FC computing power is higher and is in reference with the number of EC units in the particular time window. 


$$
F C_{t}= \begin{cases}0, & \text { if } n_{t}=0, \\ \left.\sum_{i=0}^{n}\left(E C_{i}\right)-\sum_{i=0}^{n} \delta x_{i}\right), & \text { otherwise }\end{cases}
$$

where $F C_{t}$ is the cumulative EC power at any time instant $t$. It is equal to all $E C_{t}$ units and individual units in each section of the network considered at that particular time. The CC is the sum of all the resources available at the centralized unit which can be calculated as:

$$
C C_{t}= \begin{cases}0, & \text { if } n_{t}=0, \\ \left.\sum_{j=0}^{n}\left(F C_{j}\right)-\sum_{j=0}^{n} \delta y_{j}\right), & \text { otherwise }\end{cases}
$$

where $C C_{t}$ is the computational power of all the resources available at any centralized units, the total $F C_{t}$ and $E C_{t}$ in the network. The net computational worth of the vehicular network is the sum of all the $\mathrm{CC}, \mathrm{FC}$ and $\mathrm{EC}$ units. Let function $\tau_{i}$ return the prediction of the $\mathrm{CC}, \mathrm{FC}$ and $\mathrm{EC}$ units that provide the predicted computational occurrences in the network. The forecast network computation (FNC) is dependent on the prediction of computational nodes in all the network such that $F N C_{t}$ includes all the predictions done for the next computation network at any time $t$ and can be provided by $f\left(\bigcup_{i=0}^{n} \tau_{i}\right)$. So, the total forecast network computation is given as:

$$
\operatorname{Power}(F N C)=\operatorname{Power}\left(\bigcup_{i=0}^{n} \tau_{i}\right)
$$

If there are ' $f$ ' number of CC units, ' $g$ ' number of $\mathrm{FC}$ units and ' $h$ ' number of EC units in the network, then (6) can be written as:

$$
\begin{aligned}
\operatorname{Power}(F N C)=\sum_{k=0}^{f} C & C_{k} \\
& =\sum_{i=0}^{f} \sum_{j=0}^{g} \sum_{k=0}^{h}\left(\mathrm{EC}_{\mathrm{ijk}}-\delta y_{i j}\right)
\end{aligned}
$$

Given $T_{N W}$ is the network time, the best and worst case values of FNC can be calculated from the following equations:

$$
\begin{array}{cl}
F N C_{\text {best }}=\max \left(F N C_{t}\right), & \forall t \in T_{N W} \\
F N C_{\text {worse }}=\min \left(F N C_{t}\right), & \forall t \in T_{N W}
\end{array}
$$

\section{B. Selection of Fog Unit for Inclusion}

In the traditional vehicular computational network, a normal node computation or the EC nodes are not considered. In this case, we chose all those nodes which have minimum criterion to be a computational node. It may happen that the EC nodes are computing resources in the network, but not calculated in the overall strength of the network. So, it may happen that at a certain time, the EC unit is available in the network and in future it may not because of the availability of vehicular resources and nodes leaving the EC network. So, a certain $E C_{x}$ is selected if it is available for a certain time $t$. This will solve the problem of selecting unreliable EC node for the computational strength of the network. The $E C_{x}$ is most relative and probable for inclusion if it holds the minimum criteria function which is given as:

$$
E C_{x}>\min \left(C P_{t}\right) \mid t \in T
$$

where $\mathrm{CP}$ is the minimum computational power of any edge unit.

1) Priority of Edge/Fog/Cloud: Given that $C N$ is the computational need, $P P$ is the prediction period, $S P$ is the settling period for a network, $L$ is the latency of the communication, $C$ is the cost, $T L$ is the traffic load, PreP is the pre-processing activities (such as data filtering, transformation and clearing), $C O$ is the computation offload and $S R$ is the storage resource (cache) of the node. The priority of selecting processing capability $\left(P_{\text {Compute }}\right)$ of EC, FC or $\mathrm{CC}$ is dependent on the cost of all the parameters mentioned in (12).

$$
\begin{aligned}
P_{\text {compute }}=C N+P P & +S P+L+C \\
& +T L+P r e P+C O+S R
\end{aligned}
$$

2) Failure of Edge/Fog Computing resource: The processing capabilities of the edge and fog nodes are smaller than that of the cloud in terms of the storage and processing power of the platforms. In case the edge computing resource fail to give the required processing need, the fog node is asked to provide an alternative of the failed edge node, and if the fog node also is not able to provide the processing, then the processing load falls back to the cloud itself.

\section{Ranking of Data, Nodes AND Network}

We consider, $I D_{i}$ is the ID of the vehicle or road infrastructure node, $T_{i}$ is the message periodicity of the sensor, $P_{i}$ is the message priority of the sensor, $D_{i}$ is the deadline of the message, $L_{i}$ is the load of the network at any particular time and $D P_{i}$ are the dynamic parameters of the network that are dependent on the vehicle condition and overall system load. The set of sensor nodes covered within the vicinity of a certain road side unit (RSU) are $\mathrm{S}=\left\{s_{1}, s_{2}, s_{3}, \cdots, s_{n}\right\}$, where $s_{i}$ is the number of sensors in the vicinity. The Poisson distribution, $P(x)=\frac{e^{-\lambda} \lambda^{x}}{x !}$ is considered for the arrival and departure of the any node in the network, where $\lambda$ is the average arrival rate per time unit and $P(x)$ is the probability of exactly $x$ arrivals occurring one time period.

1) Data Priority Function: Given that $\Delta T$ is the deadline by which data must be transmitted otherwise, the data is lost, $S_{r e l}$ is the relative distance between any two nodes and $\Delta V$ is the change in velocity between the receiver and the transmitter of the data unit. Then, the priority of the data $\left(P_{D}\right)$ is a function given by $f\left(\Delta T, S_{r e l}, \Delta V\right)$ where the data priority is defined as:

$$
P_{D}=\frac{(\Delta v+1)}{\left(S_{r e l}+1\right) \times(\Delta T+1)}
$$

where $\Delta T$ and $S_{r e l}$ have an inverse relationship with the priority of the data, and acceleration has direct relation. If 
the deadline of the data is approaching faster, the priority of that content is higher. And if the distance between the sender and receiver is short, the priority of the data is higher because the probability of the accuracy of information is much higher. Since $\Delta V$ can be represented as $\Delta S / \Delta T$, then (13) can be written as:

$$
P_{D}=\frac{\left(S_{r e l}+\Delta T\right)}{\Delta T \times\left(S_{r e l}+1\right) \times(\Delta T+1)}
$$

where (14) is reduced to a function of $f\left(\Delta T, S_{r e l}, \Delta V\right)$, which is much faster to calculate than (13).

2) Content Priority Function: When the data is structured with a specific layout, it contains meaningful information and contextual knowledge depending upon the objective function. This organized data is called content. The worth or value of content varies according to the request for the particular content. Given that $X_{i}$ are the interests received at any $i_{t h}$ time and $Y_{j}$ are the total number of interests received by all nodes across the network, the content priority $\left(P_{C}\right)$ can be defined by (15).

$$
P_{C}=\frac{\sum_{i=1} \frac{X_{i}}{Y_{j}}}{t_{i}}
$$

3) Network Priority Function: Network priority depends on the sum of the deterministic network (DNW) parameter and indeterministic network (IDW) parameter. Let $\Delta C$ be the duration of the contact, QoS is the quality of the network, and $B W$ is the bandwidth of the network. $D$ is the direction of the node, $A$ is the availability of the node, and $R$ is the range of the radio. Then the network priority $\left(P_{N W}\right)$ could be calculated from (16).

$$
P_{N W}=\Delta C+Q o S+B W+D+A+R
$$

4) Cost of Network Virtual Function: Any network virtual function (NVF) is created and sustained if the costs are reasonable and justified. Let $C C$ be the computational cost of the function, $F S$ the functional scalability in the network, $I$ the integrity of the data, and $C$ the centralization or the decentralization of the functional unit. The cost of network function virtualization (NFV) $\left(C_{N F V}\right)$ can be calculated from (17):

$$
\operatorname{Cost}_{N F V}=C C+F S+I+C
$$

\section{A. Design and Complexity Analysis of Ranking Algorithm of} Nodes

The proposed ML ranking algorithm for ranking the vehicular nodes is provided in 1 . Upon initialization the relative time-stamp is set to 0 . The tensor must accumulate adequate samples to calculate the mean and standard deviation, thus, it does so for the first window size $(w)$ time stamps. The trend of varying distance is captured with the rolling (moving) average and standard deviation (SD), which produces a pair of tensors. With recursion (19) and (20), the moving average $\left(\bar{\mu}_{i, j}^{(t)}\right)$ and SD $\left(\bar{\sigma}_{i, j}^{(t)}\right)$ can be calculated in constant time $O(1)$ for each pair of vehicles $d(i, j) \mid 1 \leq i, j \leq n$.
Algorithm 1: Proposed ML Ranking Algorithm.

1 Input: $\left[d(i, j)^{(t)}\right]_{n \times n \times n}:=$ A tensor time series of varying distances of window size $w$.

$2 k_{\mu}+k_{\sigma}$ : weighting coefficients for normalized mean and SD

3 s.t. $k_{\mu}+k_{\sigma}=1, \forall k_{\mu}, k_{\sigma} \geq 0$

4 Output: $\left[r(i, j)^{(t)}\right]_{n \times n}$ : A symmetric matrix, rank of link between each pair of vehicles.

5 Initialize: Set $t:=0$.

6 if $t \geq w$ then

$7 \quad$ Go to line 19.

8 Calculate moving mean and SD for capturing the trend:

9 Set $\left[\bar{\mu}^{(t)}\right]_{n \times n}:=\mu\left\{d(i, j)^{(t-w)} \ldots d(i, j)^{(t)}\right\}$ as a rolling mean.

10 Set $\left[\bar{\sigma}^{(t)}\right]_{n \times n}:=\sigma\left\{d(i, j)^{(t-w)} \ldots d(i, j)^{(t)}\right\}$ as rolling $\mathrm{SD}$.

11 if $t \leq 2 w-1$ then

12 Go to line 19.

13 Calculate median to capture average trend:

14 Set $\left[\mu^{*}\right]_{n \times n}:=\operatorname{Median}\left\{\left[\bar{\mu}^{(t-w)}\right]_{n \times n} \ldots\left[\bar{\mu}^{(t)}\right]_{n \times n}\right\}$, median of $\bar{\mu}^{(t)}$ over $t$.

15 Set $\left[\sigma^{*}\right]_{n \times n}:=\operatorname{Median}\left\{\left[\bar{\sigma}^{(t-w)}\right]_{n \times n} \ldots\left[\bar{\sigma}^{(t)}\right]_{n \times n}\right\}$, median of $\bar{\sigma}^{(t)}$ over $t$.

16 Normalization:

$17 \operatorname{Set}\left[Z_{\mu^{*}}\right]_{n \times n}:=Z\left(\left[\mu^{*}\right]_{n \times n}\right)$

$18 \operatorname{Set}\left[Z_{\sigma^{*}}\right]_{n \times n}:=Z\left(\left[\sigma^{*}\right]_{n \times n}\right)$

19 Calculate reliability:

20 Set

$$
[r(i, j)]_{n \times n}:=\sqrt{\left\{1-\left(k_{\mu} Z_{\mu^{*}}\right)^{2}+\left\{1-\left(k_{\sigma} Z_{\sigma^{*}}\right)^{2}\right\}\right.}
$$

21 Iteration: Wait for update.

22 Set $t:=t+1$.

23 Go to line 6.

24 End.

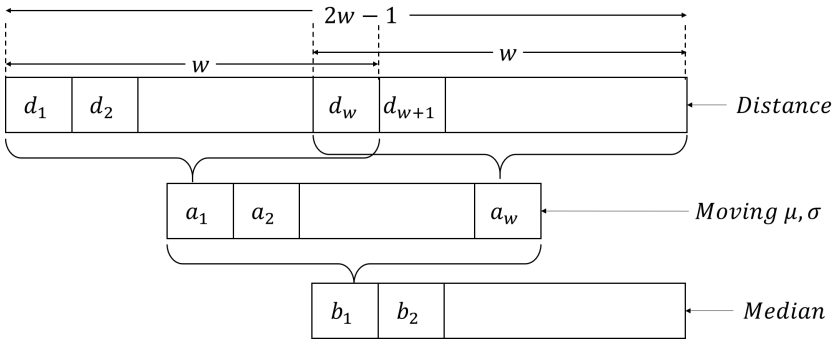

Figure 2. Sliding window to calculate moving average, SD and their median.

$$
\begin{gathered}
\mu_{(i, j)}^{(t+1)}=\frac{1}{t+1} \mu_{(i, j)}^{(t)}+d(i, j)^{(t+1)} \\
\sigma_{(i, j)}^{(t+1)}=\left[\left(\sigma_{(i, j)}^{(t)}\right)^{2}+\left(\mu_{(i, j)}^{(t)}\right)^{2}-\left(\mu_{(i, j)}^{(t+1)}\right)^{2}+\right. \\
\left.\frac{\left(d(i, j)^{(t+1))^{2}-\left(\sigma_{(i, j)}^{(t)}\right)^{2}-\left(d(i, j)^{(t)}\right)^{2}}\right.}{t+1}\right]^{1 / 2}
\end{gathered}
$$


Since each $\bar{\mu}_{i, j}^{(t)}$ and $\bar{\sigma}_{i, j}^{(t)}$ are independent, the iteration to calculate $\left[\bar{\mu}_{i, j}^{(t+1)}\right]$ and $\left[\bar{\sigma}_{i, j}^{(t+1)}\right]$ with a multi-threaded (MT) implementation would take $O(1)$ time and $O\left(n^{2}\right)$ otherwise. In the implementation, a recursive, MT approach is used to eliminate chances of any abrupt change in $\left(\bar{\mu}_{i, j}^{(t)}\right)$ and SD $\left(\bar{\sigma}_{i, j}^{(t)}\right)$ due to outliers, and transforming all $\left(\begin{array}{c}n \\ 2\end{array}\right)$ time series in to corresponding scales. Their median is taken into $\mu_{(i, j)}^{*}$ and $\sigma_{(i, j)}^{*}$, an MT implementation with 'Quick-Sort'; the step takes $O\left(w \log _{2} w\right)$ time. The median can not be calculated until $w$ number of rolling means and SD are not accumulated. To calculate the first median, the window has to slide over itself, which takes $w+w-1=2 w-1$ samples. Fig. 2 depicts how many time-stamps are required to be sampled before generating the medians.

Medians are then normalized in the next step with the help of (20) to scale in a fixed range of $[0,1]$ which takes $O(w)$ time with MT.

$$
\begin{gathered}
Z_{x}^{(t)}=\frac{x^{(t)}-\operatorname{Min}\left(\left\{x^{(t-w)} \ldots x^{(t)}\right\}\right)}{\operatorname{Max}\left(\left\{x^{(t-w)} \ldots x^{(t)}\right\}-\operatorname{Min}\left(\left\{x^{(t-w)} \ldots x^{(t)}\right\}\right.\right.} \in[0,1 \\
\text { s.t., } x \in\left\{\mu_{(i, j)}^{*}, \sigma_{(i, j)}^{*}\right\}
\end{gathered}
$$

and finally, the rank $r_{(i, j)}^{(t)}$ is calculated as the norm of $\left[\begin{array}{l}k_{\mu} \cdot Z_{\mu^{*}}^{(t)} \\ k_{\sigma} \cdot Z_{\sigma^{*}}^{(t)}\end{array}\right]$. The components are weighted by coefficients $k_{\mu}$ and $k_{\sigma}$. The rank represents, reliability of a link based on its past variations, $Z_{\mu^{*}}^{(t)} \simeq 1$ denotes the average distance between the car is increasing, which infers that the vehicles are moving further apart or the channel cost increases, hence it tends to be inferior. $Z_{\sigma^{*}}^{(t)} \simeq 1$ denotes the link is very fluctuating hence not reliable. Therefore, the reliability can be formulated as (21), which takes $O(1)$ time in MT.

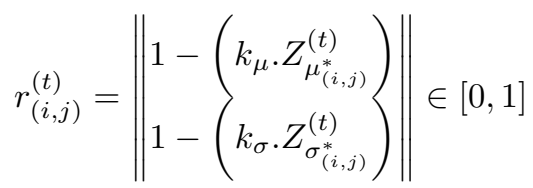

It is trivial to show that for $Z_{\mu}^{*}, Z_{\sigma}^{*}, k_{\mu}, k_{\sigma} \in[0,1]$ and $k_{\mu}+k_{\sigma}=1$ yields $r_{(i, j)}^{(t)} \in[0,1]$. Therefore with an MT implementation, the ranking with a window size of $w$ takes $O\left(w+w \log _{2} w\right)$ time.

The following section will elaborate the use of blockchain to provide distributed security to the electronic transactions in vehicular network and its usage in the proposed architecture.

\section{BlOCKChain AND ElEctronic TRANSACTIONS}

Blockchain technology is a digitized, decentralized ledger that enhances security with the help of a peer-to-peer encryption method of confirming the transactions. The need for blockchain is ever increasing due to its fundamental property of immutability and validation. Data is encrypted in the blockchain. It is possible to prove that data are altered using the signatures across all ledgers on all the nodes in the network. The main advantage of electronic commerce through vehicular mobile network is that it makes the network local to any user in an ultra-flexible and cost-effective manner; and blockchain has been used for it [53]. There are many blockchain solutions available for V2X [53]-[55]. However, these comes with stringent challenges such as placement learning for the most efficient tasks completion, coordination across multiple cars, interaction with onboard and off board computing, etc. One major concern about these is how data collection and distribution mechanisms could help build better electronic commerce systems in vehicular networks [56]. When it comes to commerce, security comes at the utmost priority. In the proposed solution, we exploit the use of blockchain to provide security, decentralized and real time information as well as transparency.

In the architecture, we adapted the micro-blockchain (MBC) from [57] to utilize in the solution. The MBC architecture helps reliability and security in vehicular networks by deployment in small regions. MBCs reduce the waiting time by small blocks to do proof of work (PoW) in small regions. Then these small MBCs combine together to produce a macro-blockchain. Minimum number of vehicles are needed to create a microblock and then these MBCs can be nested repeatedly for quick response and consensus mechanism.

Since, vehicular data and commercial vehicular transactions are important for the intelligent transportation system and these data should not be tampered. PoW is a consensus mechanism running in the Bitcoin system and it requires all the blockchain nodes to participate in the auditing process. Thus, it can be utilized to prevent vehicular data from being tampered. However, traditional blockchain systems (e.g., Bitcoin) leverage one blockchain to audit all the blocks, in which a large number of blocks have to wait for being audited and cannot be directly stored in the blockchain. Adopting low overhead consensus mechanisms can achieve lower latency in terms of storing a large number of blocks in the blockchain system. However, low overhead consensus mechanisms are typically vulnerable to malicious nodes. Therefore, in our proposed blockchain system, we reduce the waiting time by small blocks to do the proof of work (PoW) in small regions.

Vehicular data should not be tampered by malicious nodes. For permissioned blockchains, the number of blockchain nodes that participate in running a consensus mechanism is typically small. And the compromising among a few nodes can result in the tampering to the blockchain system. Comparing to public blockchains, permissioned blockchains are vulnerable to malicious nodes. Therefore, permissioned blockchains can not be considered as alternatives.

Hyperledger Fabric achieves the low latency because it leverages a small group of nodes to audit blocks. Since the number of nodes that participate in the consensus process is small, nodes in Fabric can easily compromise with each other to tamper data stored in the blockchain system. Comparing to Hyperledger Fabric, our proposed MBCs leverage the public blockchain to audit all the vehicular blocks in microblockchains. Many nodes are participating in the consensus process; thus these nodes are hard to compromise with each other to attack the blockchain system. Therefore, our proposed blockchain system is more secure than the traditional permis- 


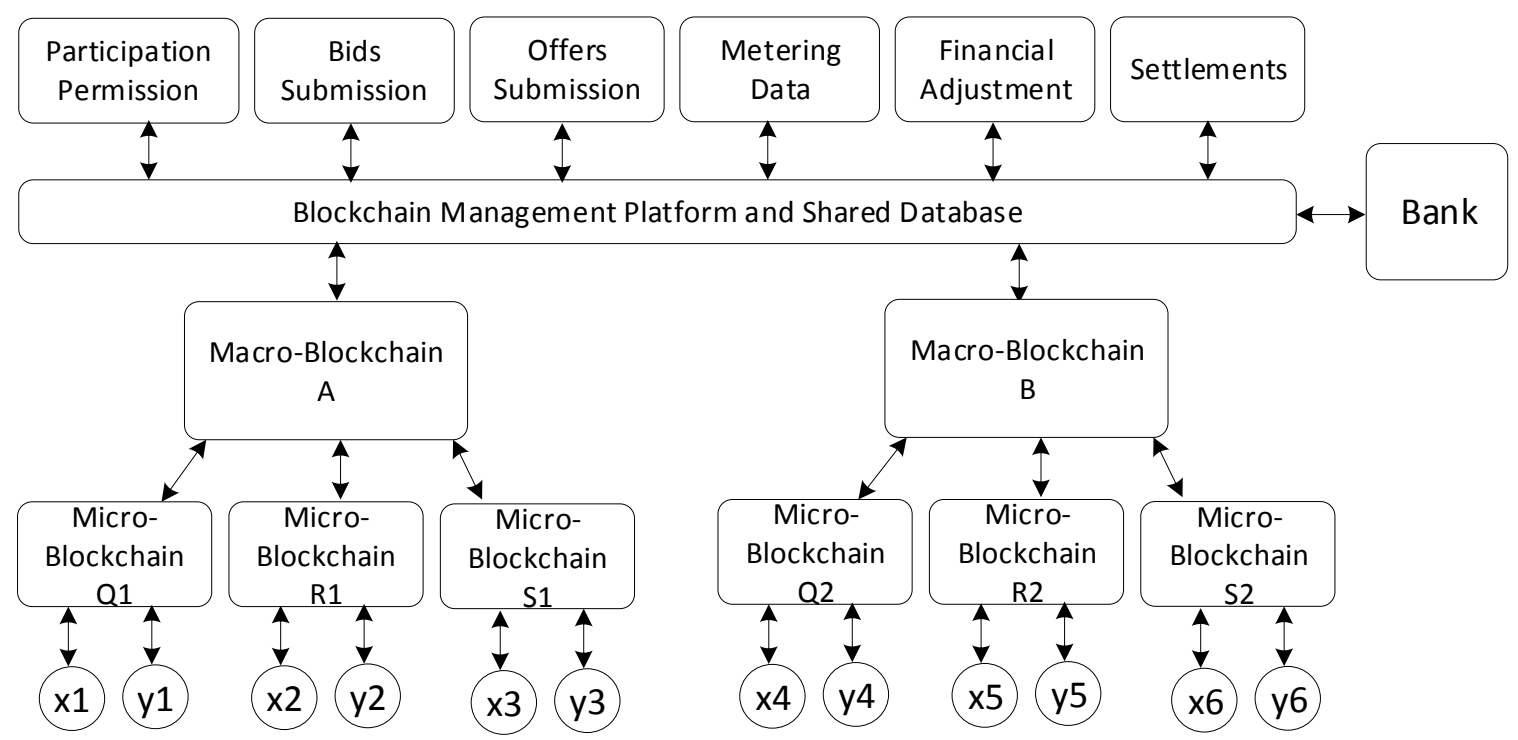

Figure 3. Micro-blockchain framework for the V2X communications.

sioned blockchain like Hyperledger Fabric.

Moreover, in this work, asymmetric cryptography is used to ensure the encryption of the data communication between any nodes in the system. After registering any bid for the transaction, two possible transactions could be done by any node in the network, such as to submit a blockchain transaction or to receive a blockchain transaction. Before doing any of the these operations, a status checkup is needed from the top of the blockchain to ensure if the existing transaction is to be followed or not.

There are different important features of any blockchain such as ledger, cryptographic hashing and Merkle trees, timestamping, consensus with fault tolerance and proof of computational work. A blockchain containing $n$ blocks, in which each successive block contains the hash of the previous block, and other parameters like transaction information, timestamp, a random number for the mining process and the details for the protocol to work.

Algorithm 2 describes how the payments of the business transaction are done. In a vehicular network, when seller $\mathrm{x}$ wants to sell its resources, it identifies itself using a blockchain address and uses a smart contract to define the terms of sale, signed with its private key. The seller provides the resources with a smart lock controlled by a smart contract. Buyer y wants to buy the resources and signs the contract with its private key. Agreeing to transfer the money from buyer blockchain address and id to blockchain address of the buyer. The smart contract is verified by each node of the blockchain network, checking if the seller is the owner of the resources and if the buyer has enough money to pay the seller. If the network agrees that the conditions are met, the buyer receives the access code for the smart lock of the smart contract. The blockchain registers the transaction and the owner of the resources to the buyer list, and the deduction of the money from the buyer account. The buyer can now utilize the resources with its private key.

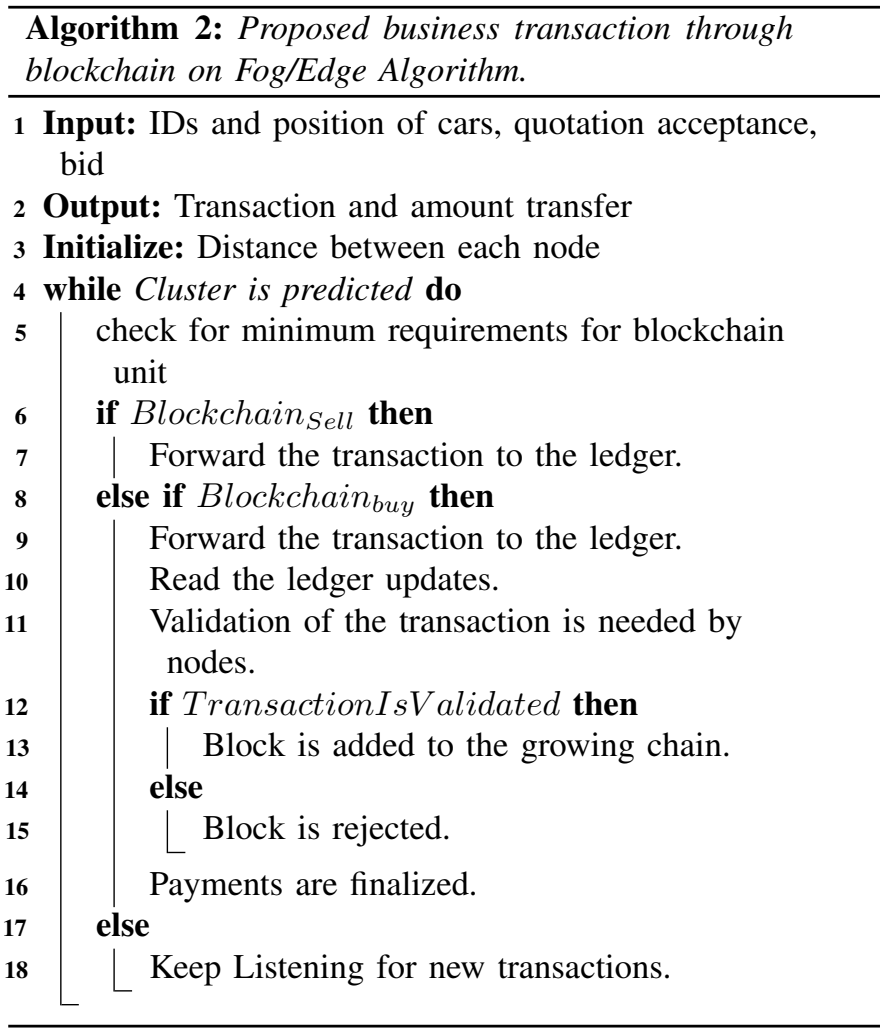

\section{CASE STUdies}

For the case studies, we adopt the proposed architecture for V2X communication in a robust, resilient and reliable manner. We chose the following two major case studies depending on the criticality of communication and massive deployment. 


\section{A. Plugin Electric Vehicles to Smart Grids Communications}

Recently, smart grids are facilitating large scale deployment of renewable energy generators, such as photovoltaic, wind power generators and Plug-In Electric Vehicles (PHEVs). In case of V2G communication, appropriate protocols are needed to communicate between the utility companies and the power generating units (PHEVs in this case). Security and privacy issues during the power injection operations among storage owners and the utility. There are three main actors in this use case, storage units, broker and utility company. Utility company wants to buy energy from PHEVs by sending power prices to the storage units for different time slots. Each storage unit masks the bid using one-time key shared with the utility. Power prices are evaluated by each vehicle power unit and prepares its bid to indicate the power to sell for the specific time slot with the announced prices. This bid communication is done between the utility company and individual storage units, but verified from the distributed nodes. Power can be purchased in both directions. To avoid the outsiders to predict and exploit the power injection, utility company and PHEVs encrypt the communication with shared keys and use blockchain to validate the bids, acceptance and payment transactions.

A decentralized ledger with multiple nodes issues public keys, which removes the threat of spoofing of digital certificates from one centralized unit. The utility company and individual storage units have digital certificates with their identity, public key, expiration date. Due to the blockchain distributed ledger, a local copy of the entire blockchain is available for the verification and no signatures need to be verified. Without the network access, hashes of the certificates can be looked up. That is why no certificate revocation list (CRLs) and online certificate status protocol (OCSP) queries are required, resulting in a better performance.

When a power purchase request for a particular time slot is made by the utility company, this request is forwarded to the individual storage nodes and verification is done for the freshness of the message using a distributed ledger. No request is allowed to be propagated inside the community if not verified by the community. In case the storage node does not accept the proposal, a no interest message is sent to the utility company. In case of accepting the bid, the request reply message includes the identities of both parties, timestamp, and masked bid. This bid is verified by the community and then the power injection from the node can be calculated. The proposed architecture can provide privacy preservation of bid value, location of node, and authenticity of power injection.

\section{B. Massive IoT data services in autonomous cars}

In autonomous driving, massive sensing is needed to ensure the safety of the vehicle, for example, adaptive electronic braking and steering systems need massive amount of sensors within a vehicle and from the environment. These sensors generate enormous data and this data is processed across different computing units for better results. For offsite migration of data and processing, higher bandwidths are required. Moreover, regular daily updates of software, new apps, and 3rd party software interactions need multiple access technologies like Wi-Fi, WAVE, and Cellular to provide cost-effective, robust and resilient communication. For the sake of reliable and secure mechanism, blockchain is used to validate the information added by any vehicle to the network. For example, an accident reported by an autonomous car needs to update the live map data across different platforms and within the sensors, and this information is validated by the blockchain shared data base.

In our daily life, each car has hundreds of sensors, computational processing units, and storage units, which can vary with the capacity of car. The computational power of any car depends on its usage, but have a specific deterministic configuration. However, when multiple CPUs from the same car or multiple CPUs of different cars collaborate to create a small edge CPU (E-CPU) to exchange the resources among them, the situation is complex. The network provides the configuration of the car to buy or sell the resources. These moving small edge units may interact with stable fixed fog and cloud units placed in the different areas of the city. The quality of the communication services, such as, standard processing, memory, bandwidth and delays are also ensured before doing any business among the cars or edge [58]. These edge and fog units are registered with the transitive cloud system through the help of blockchain based secure communication. Randomly generated pseudonym IDs are provided to the cars and the E-CPUs to provide the privacy. The IDs are provided to the transaction of the trade, so that patterns of financial transactions are intact. At any time instant, initialization settings of the market are broadcast to all the nodes involved and the local nodes access the blockchain for accepting the pseudonyms and the network settings. The application diversity of any vehicle with different resource sharing need more remote V2X communications, and for which the proposed mechanism can provide the solution.

\section{Simulation Setup}

Microscopic road traffic trajectory database helps to evaluate V2X mobility scenarios using various parameters and attributes such as vehicle coordinate and velocity. In this section, realistic vehicular traffic data is explained. Moreover, cross platform integration and experimental settings for the SDN implementation to calculate the mobility prediction are elaborated. Moreover, the deep learning engine is briefly described.

\section{A. Database}

In this work, we used a real world 6-lane highway vehicle trajectory database from the U.S. Highway 101 and the Interstate 80 (I-80) Freeway, known as the Next Generation Simulation (NGSIM) [59]. The microscopic database includes the following precise vehicle information for every $100 \mathrm{~ms}$ : vehicle ID, vehicle class (motorcycle, auto, truck), vehicle dimensions (length, width, and height), frame ID, total frames, global time, longitude and latitude, vehicle velocity, lane number (lane 1 to farthest left, lane 5 to farthest right and lane 6 as auxiliary lane), zone number, section number, and direction (in terms of left turn, right turn or straight through). 
(a)

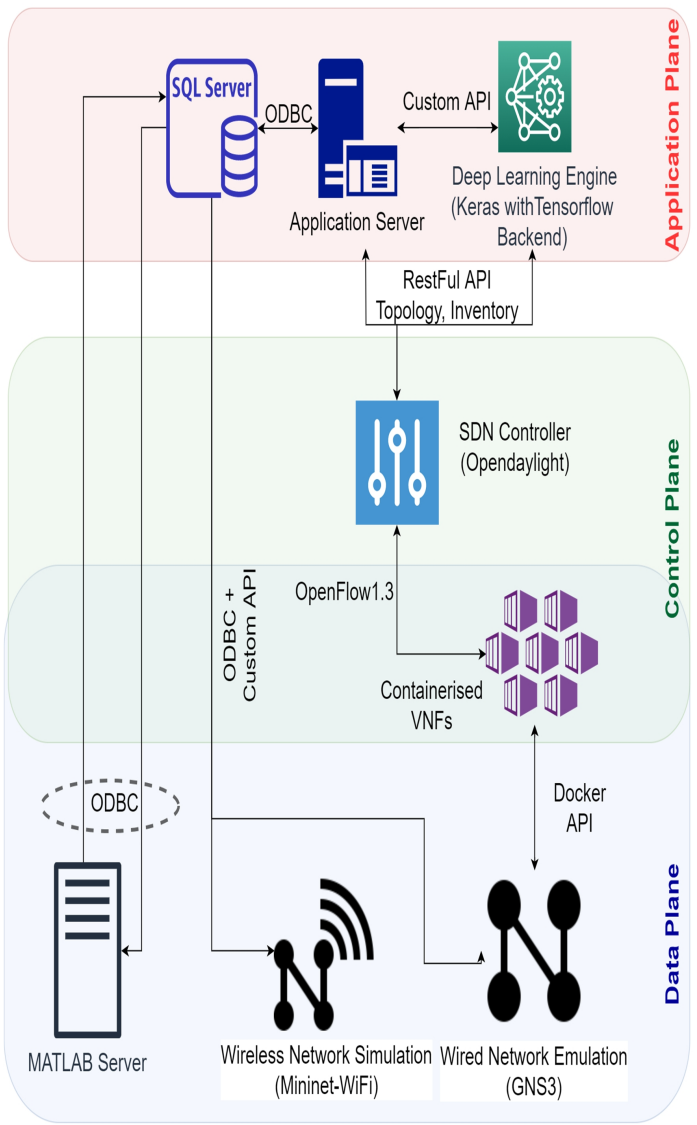

(b)

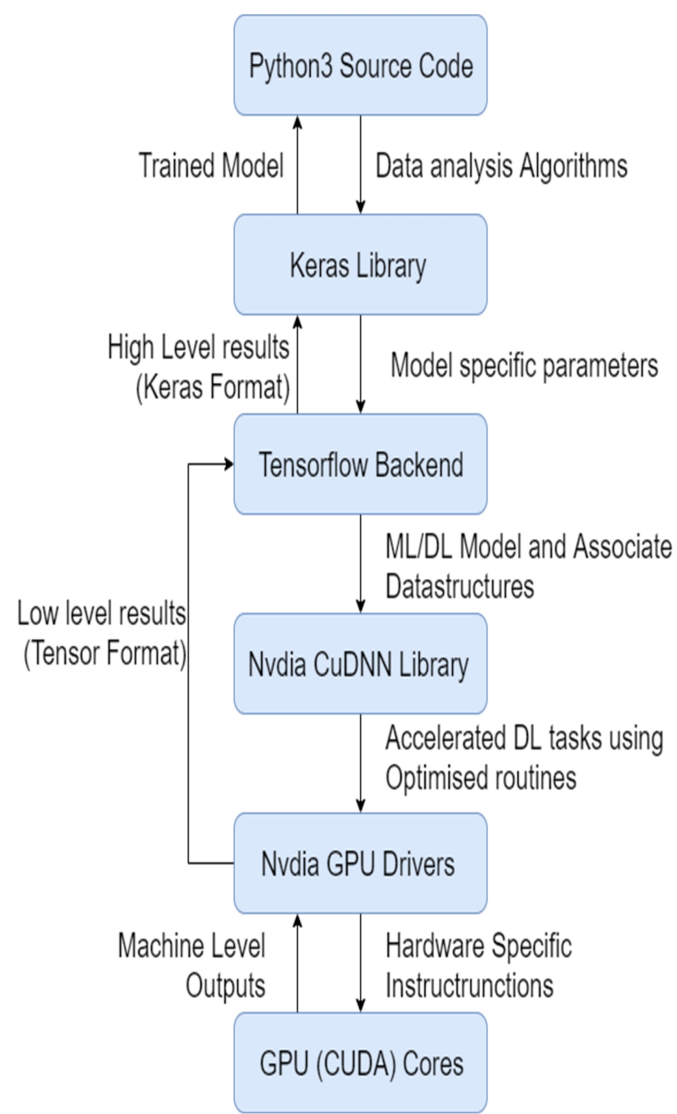

Figure 4. (a) TSLS-NLS integration framework. (b) Organization of various layers and APIs of deep learning engine with Keras and TF back-end.

\section{B. Cross Platform Integration and SDN Implementation}

Among several challenges faced while building an SDNNFV based testbed, the major challenge is the implementation and realistic nature of the results produced. A trivial problem is cross-platform interoperability, i.e. seamless integration of the system-level simulation (SLS) such as evaluation of radio and link layer parameters (SINR, path loss, radio technologies etc.) and network-level simulation/emulation (NLS) such as routing, switching, firewall policy and application layer tasks. In general, tools to perform the said tasks are specialized in nature, e.g., MATLAB for SLS and GNS3 for NLS. The SDN philosophy offers a "bird's eye" view of the network topology, regardless of the link layer technology used as shown in Fig. 4 (b). Its robust API set and abstraction features enable easy network automation and programmability. In this work, the following tools cater for the respective tasks to set up a fullfledged wireless SDN testbed.

1) MATLAB: System level simulation, evaluation of various radio parameters.

2) GNS3: A platform for network level emulation. This allows various network devices such as routers, switches, firewall, web server, etc. to be virtualised (using Qemu, Dynamips etc.) or containerized (using Docker). It also provides load-balancing by scaling the network into a cluster of multiple compute nodes. In this work, NFV is implemented by containerizing network devices (Quagga
Routers and OVS) and building networks in GNS3.

3) Mininet-wifi: Mininet-wifi provides easy building of wireless SDN. It uses MAC_80211_HWSIM drivers to simulate the Wi-Fi interfaces and provides several mobility and propagation modes. We use Mininet-wifi to implement a wireless radio interface where users are mobile and Layer 2 connectivity is provided by OVS with OpenFlow1.3.

4) Opendaylight: It is the openflow controller node that binds a number of OVSs. This integrates the radio-edge from mininet-wifi and the wired-core from GNS3, and exposes a northbound API through which an external application can read the topology and flow tables, and customised algorithms may be easily implemented to process them and results are fed back to the network. We essentially implemented a custom ranking algorithm using machine learning to reinforce the dynamic characteristics on the testbed.

5) MySQL: To build a real-time communication between MATLAB and SDN the environments, we setup a MySQL database as middleware. Although, there exists other standard alternatives such as communication over sockets, real-time unstructured databases like elastic-Search[60], however, MATLAB only supports SQL database to export runtime data using ODBC.

Fig. 4 (a) depicts the schematic illustration of integration 


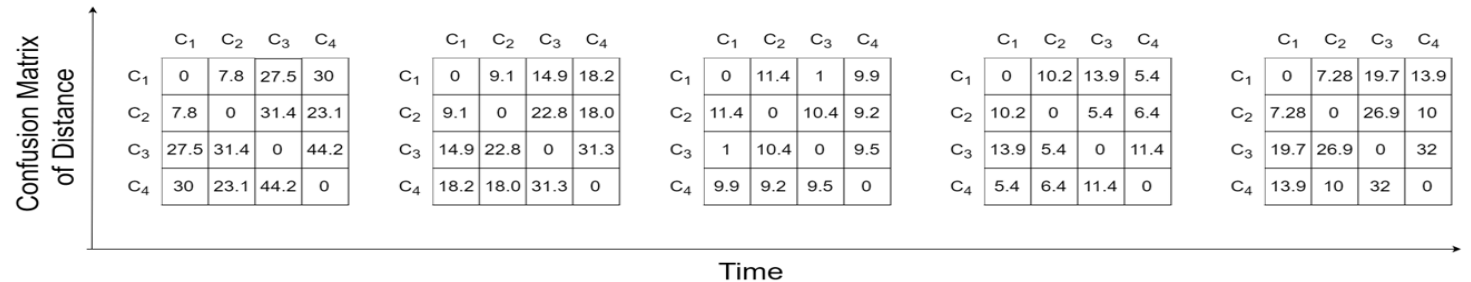

(a)

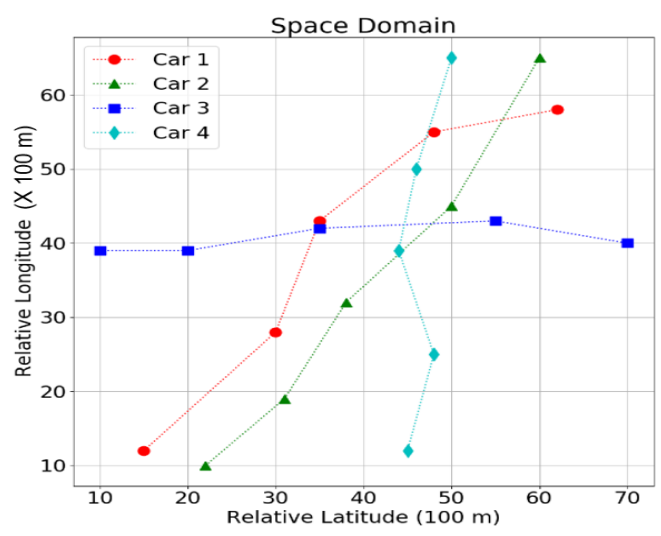

(b)

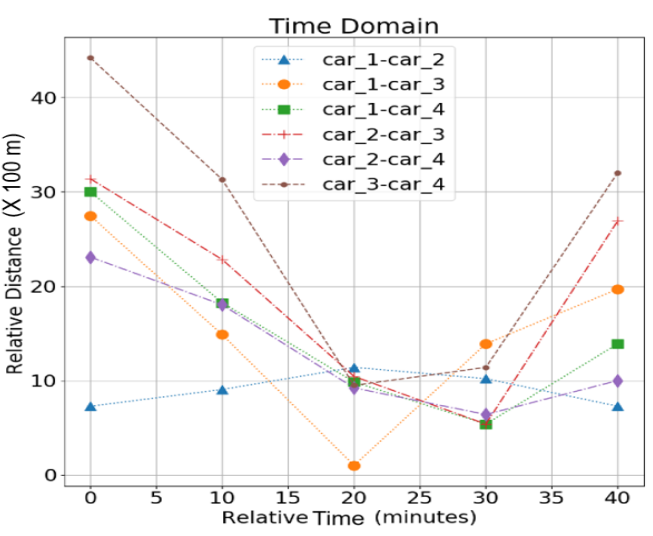

(c)

Figure 5. Time and space domain of 4 moving objects over 5 timestamps. (a) Confusion matrix of distance over time. (b) Relative distance between cars in space domain. (c) Relative distance between cars in time domain.
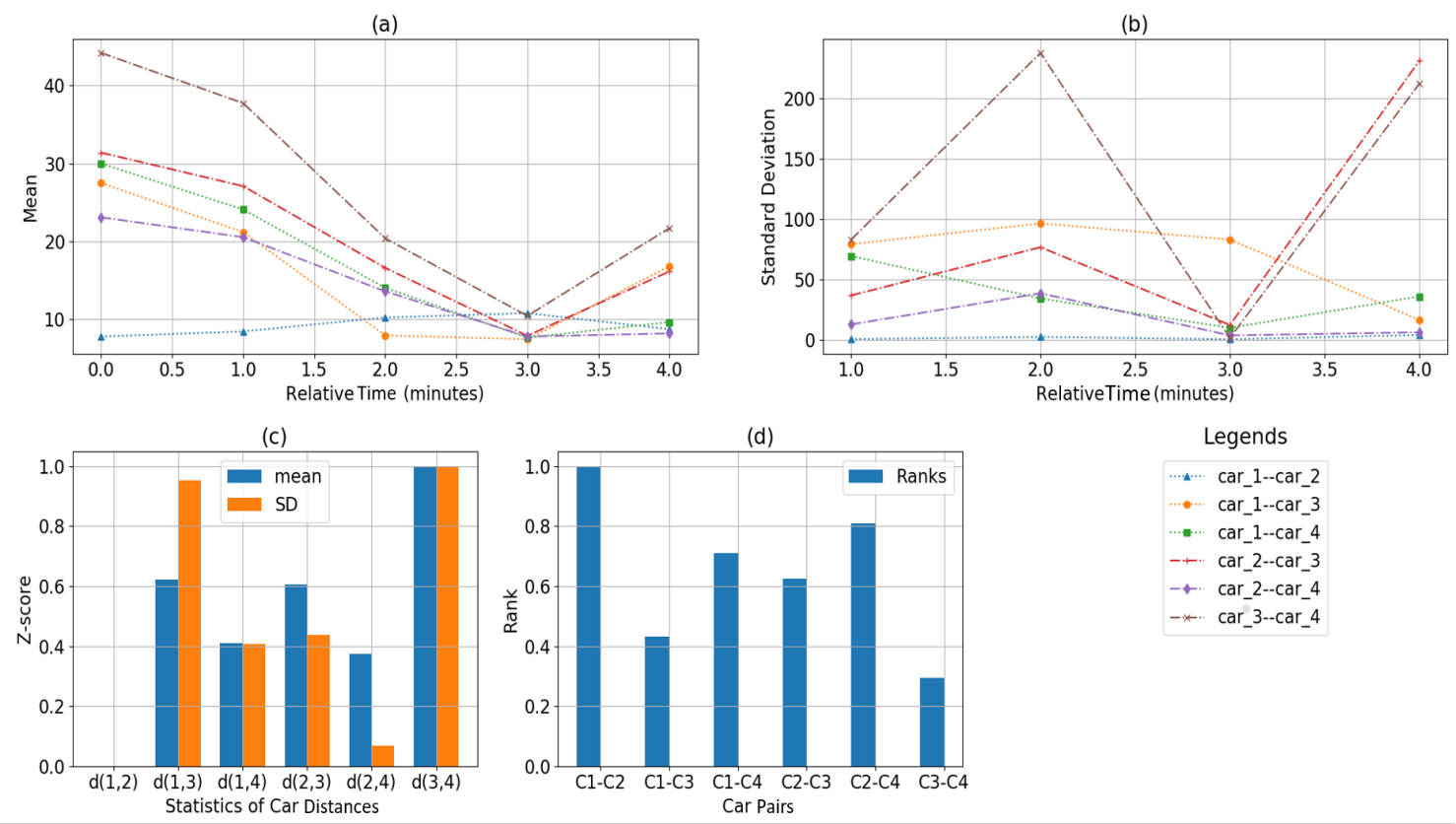

Legends

......... car_1--car_2

-.... car_1--car_3

-...... car_1--car_- car_4

-+.. car 2--car 3

-+ car_2--car_4

$-*$ car_3--car_4

Figure 6. Determining the ranking of each pair of cars in the discourse. (a) Moving average of varying distance. (b) Moving standard deviation of varying distances. (c) Z-score of median of moving mean and standard deviation. (d) Rank based on scaled mean and standard deviation. 


\begin{tabular}{|l|c|c|c|}
\hline \multirow{3}{*}{ Layers } & Type & Connection & Unit(s) \\
\cline { 2 - 4 } & Input & LSTM & 4 \\
\cline { 2 - 4 } & Hidden & Dense & 4 \\
\cline { 2 - 3 } & Output & - & 1 \\
\hline Activation & \multicolumn{3}{|c|}{ Sigmoid } \\
\hline Optimizer & \multicolumn{3}{|c|}{ ADAM } \\
\hline Loss Function & \multicolumn{2}{|c|}{ Mean Squared Error } \\
\hline \multirow{2}{*}{ Hyper-Parameters } & Batch Size & 32 \\
\cline { 2 - 3 } & Ephochs & 200 \\
\cline { 2 - 3 } & Early-Stopping & \multicolumn{2}{|c|}{ Yes } \\
\hline
\end{tabular}

Table 1

NEURAL NETWORK ARCHITECTURE PARAMETERS

between SLS and NLS within an SDN framework. Notice that the VNF containers lie along the overlapped Data and Control Planes. This is because the container clusters are further controlled by an orchestration tool such as Docker Swarm [61] or Kubernetes [62]. There are some custom APIs that are developed to establish communication between various components. The SDN Controller exposes RESTFul API on its northbound, where Inventory and Topology APIs are used to fetch flow tables and network topology information respectively.

\section{Deep Learning Engine}

As the SLS-NLS integration framework establishes a crossplatform simulation/emulation environment, the deep learning framework offers intelligence by analysing real-time network information acquired from the application server. Deep learning models are capable of resolving any hidden patterns and/or predicting sequences from historical data. The time-series of rolling mean $\left(\mu^{*}\right)$ and standard deviation $\left(\sigma^{*}\right)$ of the car-pairs $d\left(C_{i}, C_{j}\right)$ over a time-window $\mathrm{W}$ are used to calculate their corresponding rank $r(i, j)$. As the window slides over time, the varying $\left.r_{(} i, j\right)$ generates a time series. Cars that are converging to or diverging from each other produce co-relatable patterns, and cars having uncorrelated movements leaves no patterns. The deep learning (DL) model analyses these individual time series and detect their patterns. The learning problem is a timeseries analysis (TSA) problem with a learning agent, such as, a RNN with long short-term memory (LSTM). The agent learns mobility patterns to predict the possible reliability of a car-pair based on their historical data. A car-par having a high-degree of correlation results batter prediction. The agents have to learn the mobility patterns and the optimization function controls the convergence of the learning algorithm.

Fig. 4 (a) depicts the detailed organisation of various layers and their interfaces that builds a Deep learning Engine. In this approach, we opt for a Python3 based development of Deeplearning models, using Keras [63] v2.3 as a high level API with Tensorflow [64] v2.0 backend. To accelerate the learning process, Nvidia RTX2070 GPU with CuDNN library is used.

\section{RESUlts AND Discussion}

1) Data analysis and Ranking: Deep neural network (DNN) are broadly classified into two special categories based on their usage, namely: convolution neural networks(CNN) [65] and RNN, typically used for image processing and time series prediction problems, respectively [66]. Based on the type of problem being dealt with, it is trivial to classify it as a time-series prediction problem, hence RNN is chosen as a model. In the experimentation, we used the following tuning parameters as mentioned in Tab. I:

1) Layers: The DNNs consists of total of 3 layers, one input layer with four inputs $\left(k_{\mu}, k_{\sigma}, \mu^{*}, \sigma^{*}\right)$, one hidden layer with four neurons (selected by trials), and one output layer with 1 neuron that returns the predicted rank.

2) Activation: The activation function is sigmoid $\left[\frac{1}{1+e^{-x}}\right] \in[0,1]$ that bounds the rank within $[0,1]$

3) Optimizer: ADAM [67] is a stochastic gradient descent algorithm, optimal for fast convergence.

4) Loss function: The mean square error (MSE) $\epsilon=$ $\frac{1}{n} \sum_{i=1}^{n}(\hat{y}-y)^{2}$ is the heuristic that guides the Gradient Descent to converge.

5) Hyper-parameters:

Batch Size: Number of samples randomly picked from the training set at an iteration during the back propagation. Epoch: Number of times back-propagation runs to tune the parameters to its optima.

Early Stopping: This feature stops the learning process one the loss-function converges, hence producing a result of identical quality without having to iterate for all the epochs.

Fig. 5 depicts a small scale representation of the problem. For the sake of simplicity, the universe is assumed to be consisting of 4 cars and their mobility is captured for 5 timestamps. Fig. 5(a) shows the transformation of the confusion matrix, Fig. 5(b) and Fig. 5(c) illustrates the space and time domain of the matrix transformation. Hence, the problem is to approximate the distributions for a given pair of cars.

Fig. 6 depicts the ranking process in four steps; we propose a Weighted Moving Average based ranking scheme. The following describes the ranking process.

1) For each pair of cars, generate a vector $d(i, j)^{(t)}$ containing a time series of varying distance between them.

2) Calculate the moving average and standard deviation vectors $\left(\bar{\mu}^{(t)}, \bar{\sigma}^{(t)}\right)$ from $d(i, j)^{(t)}$ for a given window size (we considered 2 for capturing successive changes) and a non-zero period (Fig. 6(a) and Fig. 6(b)).

3) Calculate the Median of $\bar{\mu}^{(t)}$ and $\bar{\sigma}^{(t)}$, and perform zTransformation to scale them within $[0,1]$; let them be $\mu^{*}$ and $\sigma^{*}$ (Fig. 6(c)).

4) The rank $r_{i, j}$ is defined as $r_{i, j}=$ $\sqrt{1-\left(\left(k_{\mu} \mu^{*}\right)^{2}+\left(k_{\sigma} \sigma^{*}\right)^{2}\right)}$ (Fig. 6(d)).

The rank is high if the time series is less fluctuating and median distance is low, hence it is inversely proportional to both the mean and SD. $k_{\mu}$ and $k_{\sigma}$ are coefficient of $\mu$ and $\sigma$ respectively such that $k_{\mu}+k_{\sigma}=1$.

2) Recurrent Neural Network and Time series Prediction:

After several trials, the hyper-parameters provided in Tab. I are found to be optimal for Neural network design. Reliability variation is captured in a time-series and used for training the network, with the use of multi-threading, the training is made parallel. To accelerate the training, the Early-Stopping feature 
(a)
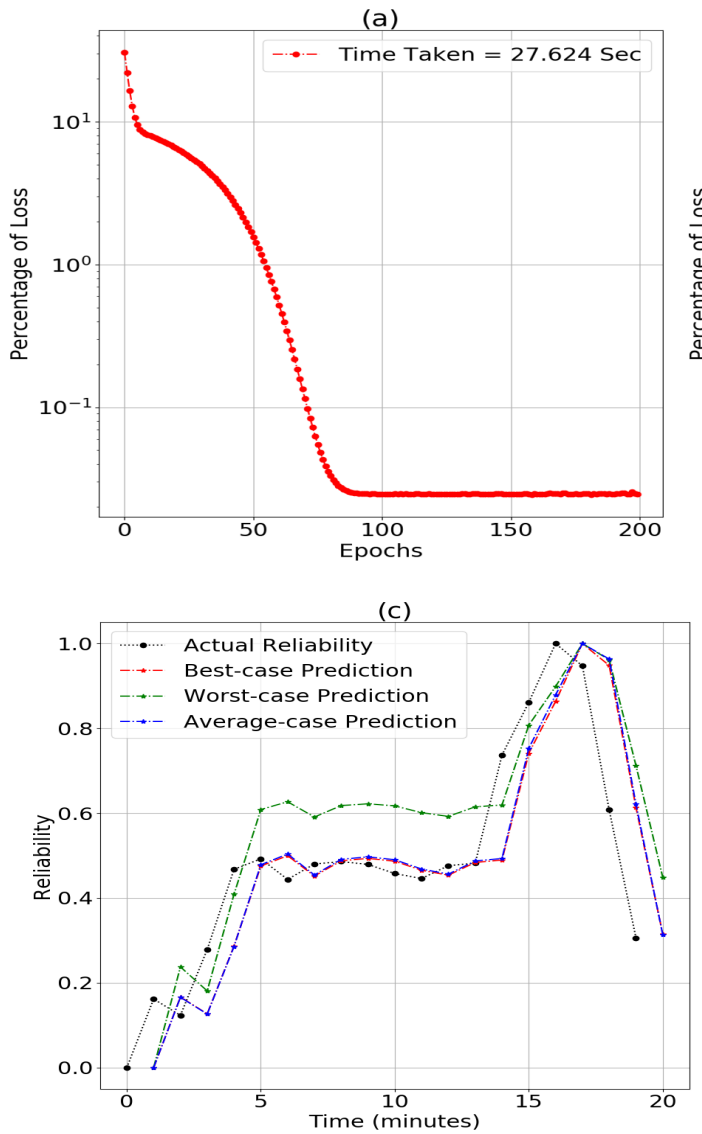

(b)

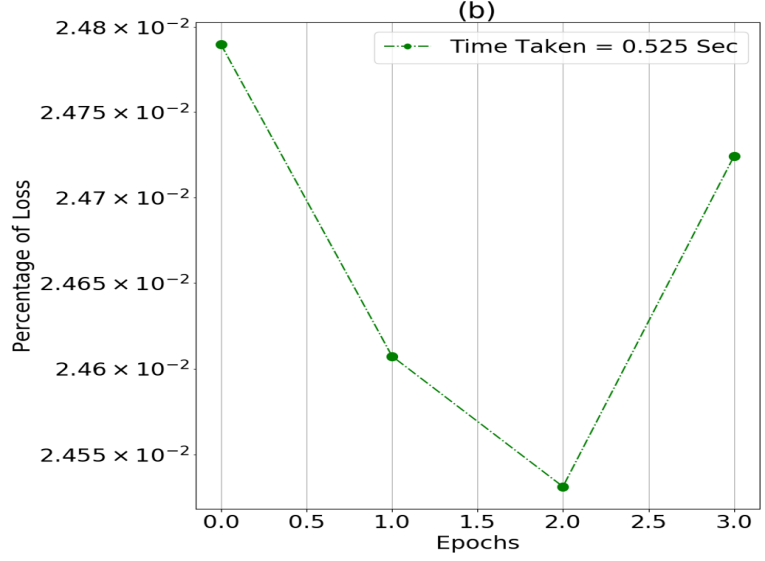

(d)

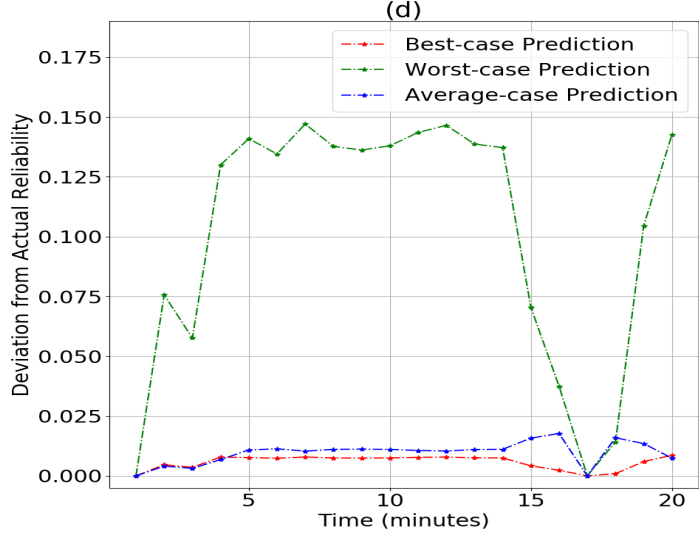

Figure 7. Determining the Ranking of each pair of cars in the discourse. (a) Loss without early stopping. (b) Loss with early stopping. (c) Comparing accuracy with respect to reliability of system. (d) Comparing accuracy with respect to deviation from actual reliability.

of Keras library is used which stops the fitting process once the loss converges (Fig. 7(a) and Fig. 7(b) depict the effect, i.e. a $98 \%$ acceleration for the subjected example). Once trained, the RNN starts predicting the reliability time series, which predicts the car pairs having higher probability to maintain high reliability which further yields a less-fluctuating distance. Fig. 7(c) depicts the comparison on the actual and predicted reliability time series of an arbitrary car pair over a common time domain. Fig. 7(d) shows the deviation from the actual reliability over time. It is evident that they are out of scale, but the predicted time series captures the pattern of actual one successfully.

3) Blockchain analysis: In terms of auditing vehicular data blocks, the micro-blockchain structure is more efficient than the traditional public blockchain structure for two reasons. Specifically, since micro-blockchains will be audited by the corresponding macro-blockchain, micro-blockchains running lightweight consensus mechanisms (e.g., proof-of-stake) can be employed to quickly audit vehicular data blocks. Moreover, in each area, multiple micro-blockchains are employed to parallelly audit the vehicular data blocks in each area. To compare public blockchain structure to micro-blockchain structure, we employ java to simulate micro-blockchain A, public blockchain B, and public blockchain $\mathrm{C}$. The difficulty levels of public blockchain B and public blockchain $\mathrm{C}$ are set as 8 , and 4 , respectively. A higher difficulty level indicates the public blockchain needs to spend more time on solving the proof-of-work puzzle. And the simulated micro-blockchain A is consisting of micro-blockchain A-1 running PoS and micro-blockchain A-2 employing PoW with a difficulty level of 6. As shown in Fig. 8 (a), with the lightweight consensus mechanism and the ability of parallelly auditing data blocks, micro-blockchain can audit more vehicular data blocks with less time.

For nodes in a public blockchain, the auditing process is consisting of signature verification, solving the proof-ofwork puzzle, hashing the previous block, and signing the verified vehicular data block. For nodes in blockchain running Proof-of-Stake (PoS), the main time consumption to complete the vehicular data block auditing process includes signature verification, previous block hash creation, and verifying data block signing. As described in Fig. 8 (b), for both microblockchain and public blockchains, time utilized for signing are almost the same when they audit the same number of vehicular data blocks; and the signing time grows along with the number of audited vehicular data blocks. Moreover, since micro-blockchain A-1 and micro-blockchain A-2 audit blocks simultaneously, micro-blockchain A and micro-blockchain A1 spends equal time on signing data blocks. As presented in Fig. 8 (c), micro-blockchain and public blockchains spend almost the same time on hashing the previous blocks when they have audited the same number of vehicular data blocks. 

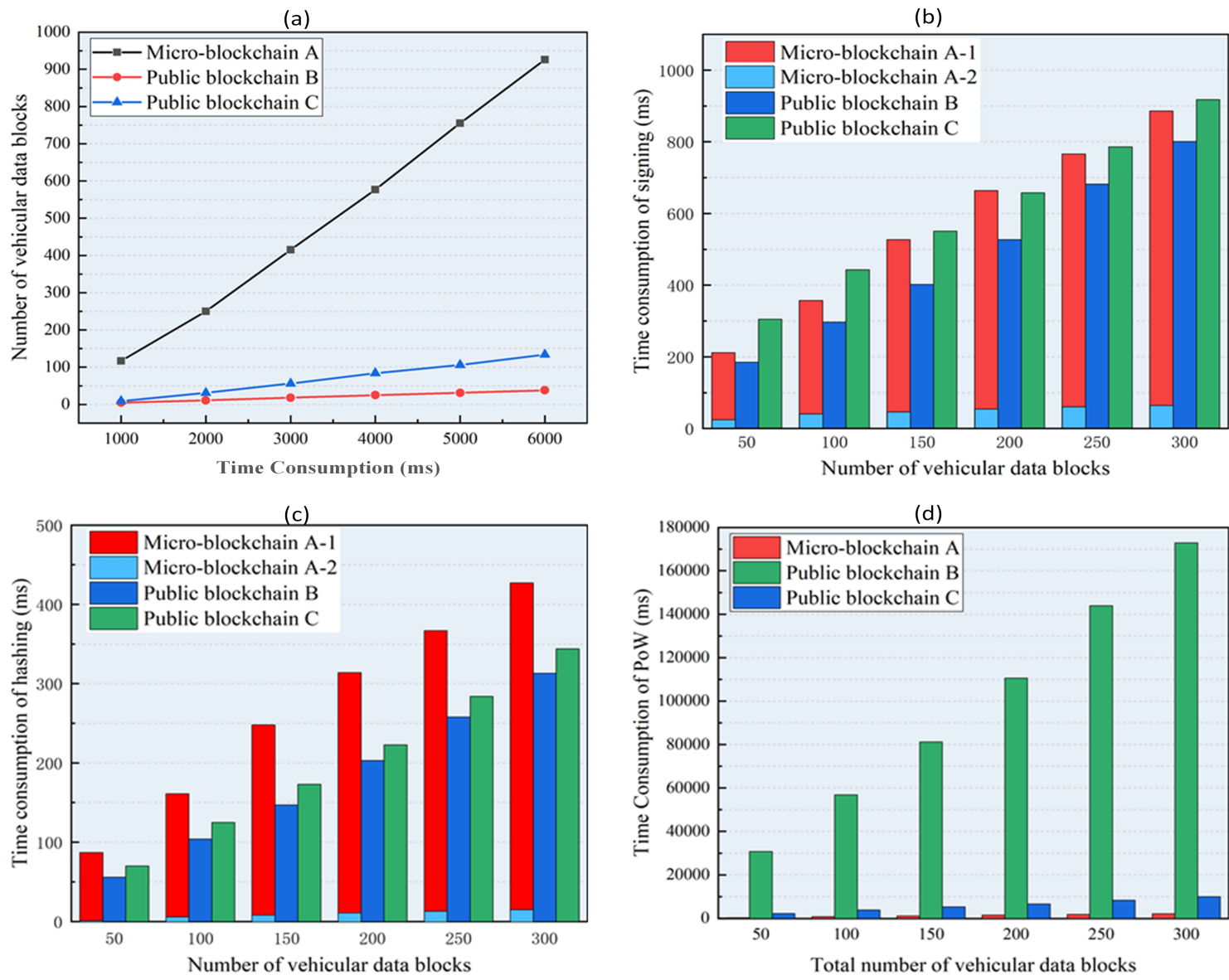

Figure 8. Proposed Micro-Blockchain parameters compared with traditional public Blockchains. (a) The relationship between time used to auditing the vehicular data blocks and the number of data blocks been audited. (b) The relationship between the number of audited vehicular data blocks and the total time used to sign the audited data blocks. (c) The relationship between the number of audited vehicular data blocks and the total time used to hash the previous blocks. (d) The relationship between the number of audited vehicular data blocks and the total time used to solve the PoW puzzle.

The blocks in the micro-blockchain are finally audited by the macro-blockchain. Therefore, micro-blockchain can leverage a low-overhead PoS consensus mechanism to quickly audit and store vehicular blocks. Moreover, the proposed blockchain system contains multiple micro-blockchains. Thus, the system simultaneously leverage these micro-blockchains to collect and store vehicular data. Fig. 8 (d) shows less time to solve the PoW puzzle when the same number of blocks are audited by the micro-blockchain compared to the public blockchains.

\section{CONCLUSions}

We have presented a holistic V2X communication architecture that addresses robustness, resilience and reliability of the network. The proposed architecture tackles the high performance vehicle communications with the help of multitechnology and multi-stage processing, such as cloud, fog and edge computing. The architecture uses deep learning tool to rank the data, nodes, and the network selection. The reliability of the network is handled using blockchain. The results showed that $98 \%$ of the time, car connectivity is predicted correctly and this eventually helps in organizing the network with multiple access technologies. The simulation results showed superiority of proposed micro-blockchain to the conventional public blockchain with different parameters, such as, signing, hashing and consumption of PoW. Since the current vehicular network scenarios are rapidly growing for autonomous cars and aerial vehicular networks, the requirement for higher data rates, low latency and reliability are needed in a cost-effective manner. In these situations, the proposed solution could make a huge impact when the potential of the proposed models are fully exploited.

\section{REFERENCES}

[1] J. P. Sterbenz, D. Hutchison, E. K. Çetinkaya, A. Jabbar, J. P. Rohrer, M. Schöller, and P. Smith, "Resilience and survivability in communication networks: Strategies, principles, and survey of disciplines," Computer Networks, vol. 54, no. 8, pp. 1245 - 1265, 2010.

[2] S. A. Busari, M. A. Khan, K. M. S. Huq, S. Mumtaz, and J. Rodriguez, "Millimetre-wave massive MIMO for cellular vehicle-to-infrastructure communication," IET Intelligent Transport Systems, vol. 13, no. 6, pp. 983-990, June 2019.

[3] E. Belyaev, A. Vinel, A. Surak, M. Gabbouj, M. Jonsson, and K. Egiazarian, "Robust vehicle-to-infrastructure video transmission for road surveillance applications," IEEE Transactions on Vehicular Technology, vol. 64, no. 7, pp. 2991-3003, July 2015.

[4] X. Huang, J. Wu, W. Li, Z. Zhang, F. Zhu, and M. Wu, "Historical spectrum sensing data mining for cognitive radio enabled vehicular ad-hoc networks," IEEE Transactions on Dependable and Secure Computing, vol. 13, no. 1, pp. 59-70, Jan 2016.

[5] N. Lyamin, A. Vinel, M. Jonsson, and B. Bellalta, "Cooperative awareness in VANETs: On ETSI EN 302 637-2 Performance," IEEE Transactions on Vehicular Technology, vol. 67, no. 1, pp. 17-28, Jan 2018. 
[6] M. A. Masrur, A. G. Skowronska, J. Hancock, S. W. Kolhoff, D. Z. McGrew, J. C. Vandiver, and J. Gatherer, "Military-based vehicle-to-grid and vehicle-to-vehicle microgrid-system architecture and implementation," IEEE Transactions on Transportation Electrification, vol. 4, no. 1, pp. 157-171, March 2018.

[7] H. Ko, S. Pack, and V. C. M. Leung, "Mobility-aware vehicle-togrid control algorithm in microgrids," IEEE Transactions on Intelligent Transportation Systems, vol. 19, no. 7, pp. 2165-2174, July 2018.

[8] H. Turker and S. Bacha, "Optimal minimization of plug-in electric vehicle charging cost with vehicle-to-home and vehicle-to-grid concepts," IEEE Transactions on Vehicular Technology, vol. 67, no. 11, pp. 10281 10292 , Nov 2018.

[9] W. Wang, Y. Jiang, and W. Wu, "Multiagent-based resource allocation for energy minimization in cloud computing systems," IEEE Transactions on Systems, Man, and Cybernetics: Systems, vol. 47, no. 2, pp. 205-220, Feb 2017.

[10] "IEEE standard for information technology-Telecommunications and information exchange between systems local and metropolitan area networks-specific requirements - part 11: Wireless lan medium access control (mac) and physical layer (phy) specifications," IEEE Std 802.112016 (Revision of IEEE Std 802.11-2012), pp. 1-3534, Dec 2016.

[11] "IEEE standard for information technology-telecommunications and information exchange between systems local and metropolitan area networks-specific requirements part 11: Wireless lan medium access control (mac) and physical layer (phy) specifications," IEEE Std 802.112012 (Revision of IEEE Std 802.11-2007), pp. 1-2793, March 2012.

[12] K. Wang, H. Yin, W. Quan, and G. Min, "Enabling collaborative edge computing for software defined vehicular networks," IEEE Network, vol. 32, no. 5, pp. 112-117, Sep. 2018.

[13] A. Ahmad, A. Paul, M. Khan, S. Jabbar, M. M. U. Rathore, N. Chilamkurti, and N. Min-Allah, "Energy efficient hierarchical resource management for mobile cloud computing," IEEE Transactions on Sustainable Computing, vol. 2, no. 2, pp. 100-112, April 2017.

[14] J. Wang, Y. Shao, Y. Ge, and R. Yu, "A survey of vehicle to everything (v2x) testing," Sensors, vol. 19, no. 2, p. 334, 2019.

[15] Z. MacHardy, A. Khan, K. Obana, and S. Iwashina, "V2x access technologies: Regulation, research, and remaining challenges," IEEE Communications Surveys \& Tutorials, vol. 20, no. 3, pp. 1858-1877, 2018.

[16] J. Heo, B. Kang, J. M. Yang, J. Paek, and S. Bahk, "Performance-cost tradeoff of using mobile roadside units for v2x communication," IEEE Transactions on Vehicular Technology, vol. 68, no. 9, pp. 9049-9059, 2019.

[17] S. Husain, A. Kunz, A. Prasad, E. Pateromichelakis, and K. Samdanis, "Ultra-high reliable $5 \mathrm{~g}$ v2x communications," IEEE Communications Standards Magazine, vol. 3, 062019.

[18] A. Kostopoulos, I. Chochliouros, J. Ferragut, Y. Ma, M. Kutila A. Gavras, S. Horsmanheimo, K. Zhang, L. Ladid, A. Dardamanis, and M. Kourtis, "Use cases and standardisation activities for embb and $\mathrm{v} 2 \mathrm{x}$ scenarios," in 2020 IEEE International Conference on Communications Workshops (ICC Workshops), 2020, pp. 1-7.

[19] M. A. Khan, S. Sargento, and M. Luis, "Data collection from smart-city sensors through large-scale urban vehicular networks," in 2017 IEEE 86th Vehicular Technology Conference (VTC-Fall), 2017, pp. 1-6.

[20] M. Awais, H. S. Khaliq, and W. T. Khan, "A novel dual-band millimeterwave antenna for automotive radar and multi-gigabit wireless communications," in 2017 Progress in Electromagnetics Research Symposium - Fall (PIERS - FALL), 2017, pp. 2802-2807.

[21] A. K. Ligo and J. M. Peha, "Spectrum for v2x: Allocation and sharing," IEEE Transactions on Cognitive Communications and Networking, vol. 5, no. 3, pp. 768-779, 2019.

[22] G. P. W. N.B.A, J. Haapola, and T. Samarasinghe, "A discrete-time markov chain based comparison of the mac layer performance of c-v2x mode 4 and ieee 802.11p," IEEE Transactions on Communications, pp. $1-1,2020$

[23] M. A. Khan, A. Jadoon, K. M. S. Haq, S. Mumtaz, and J. Rodrigues, "An overview of resilient and automatic model-based testing approaches for automotive industry," in 2019 IEEE International Conference on Communications Workshops (ICC Workshops), 2019, pp. 1-6.

[24] S. H. Bouk, S. H. Ahmed, R. Hussain, and Y. Eun, "Named data networking's intrinsic cyber-resilience for vehicular cps," IEEE Access, vol. 6, pp. 60570-60585, 2018.

[25] S. Husain, A. Kunz, A. Prasad, E. Pateromichelakis, K. Samdanis, and J. Song, "The road to $5 \mathrm{~g}$ v2x: Ultra-high reliable communications," in 2018 IEEE Conference on Standards for Communications and Networking (CSCN), 2018, pp. 1-6.
[26] A. Khan, J. Almeida, B. Fernandes, M. Alam, P. Pedreiras, and J. Ferreira, "Towards reliable wireless vehicular communications," in 2015 IEEE 18th International Conference on Intelligent Transportation Systems, 2015, pp. 167-172.

[27] Y. Wang, J. Zheng, and N. Mitton, "Delivery delay analysis for roadside unit deployment in vehicular ad hoc networks with intermittent connectivity," IEEE Transactions on Vehicular Technology, vol. 65, no. 10, pp. 8591-8602, 2016

[28] K. Abboud, H. A. Omar, and W. Zhuang, "Interworking of DSRC and cellular network technologies for V2X communications: A survey," IEEE Transactions on Vehicular Technology, vol. 65, no. 12, pp. 94579470, Dec 2016.

[29] Q. Zhang, H. Zheng, J. Lan, J. An, and H. Peng, "An autonomous information collection and dissemination model for large-scale urban road networks," IEEE Transactions on Intelligent Transportation Systems, vol. 17, no. 4, pp. 1085-1095, April 2016.

[30] C. Chen, K. Li, S. G. Teo, X. Zou, K. Li, and Z. Zeng, "Citywide traffic flow prediction based on multiple gated spatio-temporal convolutional neural networks," ACM Trans. Knowl. Discov. Data, vol. 14, no. 4, May 2020. [Online]. Available: https://doi.org/10.1145/3385414

[31] P. Kachroo, N. Shlayan, S. Roy, and M. Zhang, "High-performance vehicle streams: Communication and control architecture," IEEE Transactions on Vehicular Technology, vol. 63, no. 8, pp. 3560-3568, Oct 2014.

[32] J. Chen, K. Li, Q. Deng, K. Li, and P. S. Yu, "Distributed deep learning model for intelligent video surveillance systems with edge computing," IEEE Transactions on Industrial Informatics, pp. 1-1, 2019.

[33] A. A. Visheratin, M. Melnik, and D. Nasonov, "Workflow scheduling algorithms for hard-deadline constrained cloud environments," Procedia Computer Science, vol. 80, pp. 2098 - 2106, 2016, international Conference on Computational Science 2016, ICCS 2016, 6-8 June 2016, San Diego, California, USA. [Online]. Available: http: //www.sciencedirect.com/science/article/pii/S1877050916310195

[34] S. Liao, J. Li, J. Wu, W. Yang, and Z. Guan, "Fog-enabled vehicle as a service for computing geographical migration in smart cities," IEEE Access, vol. 7, pp. 8726-8736, 2019.

[35] S. A. Busari, K. M. S. Huq, S. Mumtaz, J. Rodriguez, Y. Fang, D. C. Sicker, S. Al-Rubaye, and A. Tsourdos, "Generalized hybrid beamforming for vehicular connectivity using thz massive mimo," IEEE Transactions on Vehicular Technology, vol. 68, no. 9, pp. 8372-8383, Sep. 2019

[36] K. Okafor, I. Achumba, and G. Ononiwu, "Leveraging fog computing for scalable iot datacenter using spine-leaf network topology," Journal of Electrical and Computer Engineering, vol. 2017, pp. 1-11, 042017.

[37] E. C. Anderson, K. C. Okafor, O. Nkwachukwu, and D. O. Dike, "Real time car parking system: A novel taxonomy for integrated vehicular computing," in 2017 International Conference on Computing Networking and Informatics (ICCNI), 2017, pp. 1-9.

[38] X. Kong, F. Xia, Z. Ning, A. Rahim, Y. Cai, Z. Gao, and J. Ma, "Mobility dataset generation for vehicular social networks based on floating car data," IEEE Transactions on Vehicular Technology, vol. 67, no. 5, pp. 3874-3886, May 2018.

[39] S. Kokogias, L. Svensson, G. Collares Pereira, R. Oliveira, X. Zhang, $X$. Song, and J. Mårtensson, "Development of platform-independent system for cooperative automated driving evaluated in gcdc 2016," IEEE Transactions on Intelligent Transportation Systems, vol. 19, no. 4, pp. 1277-1289, April 2018

[40] W. Liu and Y. Shoji, "Edge-assisted vehicle mobility prediction to support v2x communications," IEEE Transactions on Vehicular Technology, vol. 68, no. 10, pp. 10227-10238, Oct 2019.

[41] P. Themann, J. Bock, and L. Eckstein, "Optimisation of energy efficiency based on average driving behaviour and driver's preferences for automated driving," IET intelligent transport systems, vol. 9, no. 1, pp. $50-58,2014$.

[42] K. C. Okafor, G. C. Ononiwu, S. Goundar, V. C. Chijindu, and C. C. Udeze, "Towards complex dynamic fog network orchestration using embedded neural switch," International Journal of Computers and Applications, vol. 0, no. 0, pp. 1-18, 2018. [Online]. Available: https://doi.org/10.1080/1206212X.2018.1517440

[43] D. R. McNeil, "A solution to the fixed-cycle traffic light problem for compound poisson arrivals," Journal of Applied Probability, vol. 5, no. 3, pp. 624-635, 1968. [Online]. Available: http: //www.jstor.org/stable/3211926

[44] K. Wüst and A. Gervais, "Do you need a blockchain?" in 2018 Crypto Valley Conference on Blockchain Technology (CVCBT), 2018, pp. 45-54.

[45] D. B. Rawat, R. Doku, A. Adebayo, C. Bajracharya, and C. Kamhoua, "Blockchain enabled named data networking for secure vehicle-to- 
everything communications," IEEE Network, vol. 34, no. 5, pp. 185-189, 2020.

[46] K. J. Ahmed and M. J. Lee, "Secure LTE-based V2X service," IEEE Internet of Things Journal, vol. 5, no. 5, pp. 3724-3732, Oct 2018

[47] Z. Ning, X. Wang, and J. Huang, "Mobile edge computing-enabled $5 \mathrm{~g}$ vehicular networks: Toward the integration of communication and computing," IEEE Vehicular Technology Magazine, vol. 14, no. 1, pp. 54-61, 2019.

[48] X. Wang, Z. Ning, and L. Wang, "Offloading in internet of vehicles: A fog-enabled real-time traffic management system," IEEE Transactions on Industrial Informatics, vol. 14, no. 10, pp. 4568-4578, 2018.

[49] M. Giordani, T. Shimizu, A. Zanella, T. Higuchi, O. Altintas, and M. Zorzi, "Path loss models for v2v mmwave communication: Performance evaluation and open challenges," in 2019 IEEE 2nd Connected and Automated Vehicles Symposium (CAVS), 2019, pp. 1-5.

[50] S. Sun, T. S. Rappaport, M. Shafi, P. Tang, J. Zhang, and P. J. Smith, "Propagation models and performance evaluation for $5 \mathrm{~g}$ millimeter-wave bands," IEEE Transactions on Vehicular Technology, vol. 67, no. 9, pp. $8422-8439,2018$

[51] 3GPP, "5G; Study on channel model for frequencies from 0.5 to 100 GHz," 3rd Generation Partnership Project (3GPP), Technical Specification (TS), 05 2017, version 14.0.0.

[52] V. V. Chetlur and H. S. Dhillon, "Coverage and rate analysis of downlink cellular vehicle-to-everything (c-v2x) communication," IEEE Transactions on Wireless Communications, vol. 19, no. 3, pp. 17381753,2020

[53] G. S. Aujla, A. Singh, M. Singh, S. Sharma, N. Kumar, and K. R. Choo, "Blocked: Blockchain-based secure data processing framework in edge envisioned v2x environment," IEEE Transactions on Vehicular Technology, vol. 69, no. 6, pp. 5850-5863, 2020.

[54] F. Jameel, M. A. Javed, S. Zeadally, and R. Jäntti, "Efficient mining cluster selection for blockchain-based cellular v2x communications," IEEE Transactions on Intelligent Transportation Systems, pp. 1-9, 2020.

[55] R. Jabbar, N. Fetais, M. Kharbeche, M. Krichen, K. Barkaoui, and M. Shinoy, "Blockchain for the internet of vehicles: How to use blockchain to secure vehicle-to-everything ( $22 \mathrm{x})$ communication and payment ?" IEEE Sensors Journal, pp. 1-1, 2021.

[56] I. Agudo, M. Montenegro-Gomez, and J. Lopez, "A blockchain approach for decentralized v2x (d-v2x)," IEEE Transactions on Vehicular Technology, pp. 1-1, 2020.

[57] H. Liang, J. Wu, S. Mumtaz, J. Li, X. Lin, and M. Wen, "Mbid: Microblockchain-based geographical dynamic intrusion detection for v2x," IEEE Communications Magazine, vol. 57, no. 10, pp. 77-83, Oct. 2019.

[58] "IEEE standard for information technology-telecommunications and information exchange between systems local and metropolitan area networks-specific requirements part 11: Wireless lan medium access control (mac) and physical layer (phy) specifications amendment 3: Enhancements for very high throughput to support chinese millimeter wave frequency bands (60 ghz and $45 \mathrm{ghz}$ )," IEEE Std 802.11aj-2018, pp. 1-306, April 2018.

[59] ITS Data Hub, U.S. Department of Transportation Federal Highway Administration, "Next Generation Simulation (NGSIM) vehicle trajectories and supporting data," Nov. 2019.

[60] Elastic, "Elasticsearch reference," Nov. 2020. [Online]. Available: www.elastic.co/guide/en/elasticsearch/reference/current/index.html

[61] Docker Documentation, "Swarm mode overview," Nov. 2020. [Online]. Available: www.docs.docker.com/engine/swarm

[62] Kubernetes.io, "Concepts," Nov. 2020. [Online]. Available: kubernetes. io/docs/concepts

[63] Keras, "Keras," Nov. 2020. [Online]. Available: keras.io

[64] TensorFlow, "All symbols in tensorflow 2.0," Nov. 2020. [Online]. Available: www.tensorflow.org/api_docs/python

[65] Jun Zhang and K. F. Man, "Time series prediction using rnn in multi-dimension embedding phase space," in SMC'98 Conference Proceedings. 1998 IEEE International Conference on Systems, Man, and Cybernetics (Cat. No.98CH36218), vol. 2, Oct. 1998, pp. 1868-1873 vol.2.

[66] X. Gao and H. Xiong, "A hybrid wavelet convolution network with sparse-coding for image super-resolution," in 2016 IEEE International Conference on Image Processing (ICIP), Sep. 2016.

[67] D. P. Kingma and J. Ba, "Adam: A method for stochastic optimization," in 3rd International Conference for Learning Representations, San Diego, 2015.

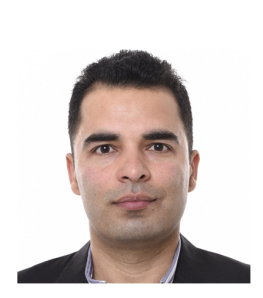

Muhammad Awais Khan is a research engineer at Instituto de Telecomunicações, Aveiro Portugal. He received his MS in Information and Communication Systems Securities from Royal Institute of Technology (KTH), Stockholm, Sweden. He actively participated in multiple European Commission projects, such as FP7 project: Intelligent Cooperative Sensing for Improved Traffic Efficiency (ICSI), Horizon 2020 project: Sensing and Serving a Moving City, SWARMS, Semi 40, THz-BEGUN, and 5GPP5GENEIS. His research work contributed to create an optimal vehicular network for provision of data services in a smart city. Currently, he is working in vehicular networks, real time systems, autonomous cars and cloud computing. His interests lie in Intelligent Transportation System (ITS), dependable vehicular communications, information security, IoT and Smart City Applications, open distributed system, their design, algorithms, architectures, and protocols.

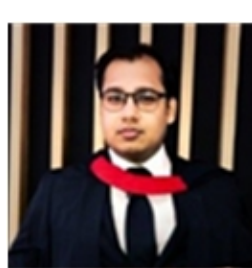

Saptarshi Ghosh obtained his M.E. in software Engineering from Jadavpur University, India and M.Sc. in Smart Networks from University of the West of Scotland, UK. He has also been a recipient of GATE and Erasmus-Mundus scholarship. Currently he is working towards his $\mathrm{PhD}$ in Computer Science \& Informatics at London South Bank University, supported by Marie-Curie fund with the research area focused in Machine Learning's application to Self Organised SDN for 5G. His area of interests includes Network programmability and automation,

Routing and Deep Reinforcement Learning.

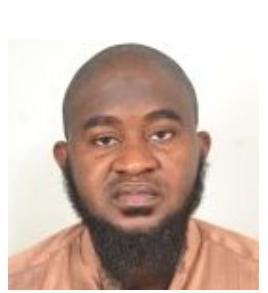

Sherif Adeshina Busari received the B.Eng. and M.Eng. degrees in electrical and electronics engineering from the Federal University of Technology Akure, Nigeria, in 2011 and 2015, respectively. He received a $\mathrm{PhD}$ in Telecommunications from the Universidade de Aveiro, Portugal in 2020 . He serves as a reviewer for many journals, and TPC member and reviewer for many conferences. His research interests include wireless channel modeling, millimeter-wave and terahertz communications, massive MIMO, intelligent transport systems, radio resource management and optimization for beyond-5G mobile networks. He is a Member of the IEEE and ComSoc, and author of more than 20 scientific publications.

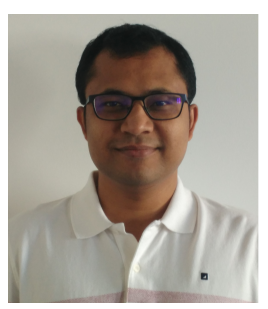

Kazi Mohammed Saidul Huq obtained his B.Sc. degree in computer science and engineering from Ahsanullah University of Science and Technology, Bangladesh, in 2003, and his M.Sc. and Ph.D. degrees in Electrical Engineering from Blekinge Institute of Technology, Sweden, in 2006 and the University of Aveiro, Portugal, in 2014, respectively. In 2008, he became a researcher at the Instituto de Telecomunicações, Aveiro, Portugal, with primary interests in $5 \mathrm{G}$. He is the recipient of the prestigious Marie-Curie Individual Fellowship for Beyond 5G research by EU. Currently, he is a Senior Research Fellow of the Faculty of Computing, Engineering, and Science at the University of South Wales, United Kingdom. He is the Associate Editor of the IET Quantum Communication Journal. 


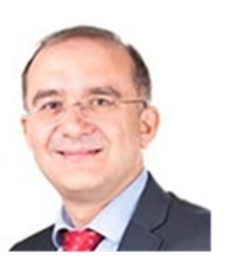

Tasos Dagiuklas is a leading researcher and expert in the fields of smart Internet technologies. He is the leader of the Smart Internet Technologies (SuITE) research group at the London South Bank University where he also acts as the Head of Cognitive Systems Reseach Centre. Tasos received the Engineering Degree from the University of Patras-Greece in 1989, the M.Sc. from the University of ManchesterUK in 1991 and the Ph.D. from the University of Essex-UK in 1995, all in Electrical Engineering. He has been a principal investigator, co-investigator, project and technical manager, coordinator and focal person of more than 20 internationally R\&D and Capacity training projects in the areas of FixedMobile Convergence, 4G/5G networking technologies, VoIP and multimedia networking. His research interests lie in the field of Systems Beyond 5G/6G networking technologies, programmable networks, Car2X communications and cyber security for smart Internet systems.

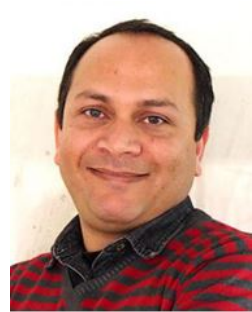

Shahid Mumtaz Shahid Mumtaz is an IET Fellow, IEEE ComSoc and ACM Distinguished speaker, recipient of IEEE ComSoC Young Researcher Award (2020), founder and EiC of IET "Journal of Quantum communication", Vice-Chair: Europe/Africa RegionIEEE ComSoc: Green Communications \& Computing society, and Vice-chair for IEEE standard on P1932.1: Standard for Licensed/Unlicensed Spectrum Interoperability in Wireless Mobile Networks. $\mathrm{He}$ is also a Senior 5G Consultant at Huawei, Sweden. He is the author of 4 technical books, 12 book chapters, $250+$ technical papers (150+ Journal/transaction, 80+ conference, 2 IEEE best paper award- in the area of mobile communications. Most of his publication is in the field of Wireless Communication. He is serving as Scientific Expert and Evaluator for various Research Funding Agencies. He was awarded an Alain Bensoussan fellowship in 2012. He is the recipient of the NSFC Researcher Fund for Young Scientist in 2017 from China.

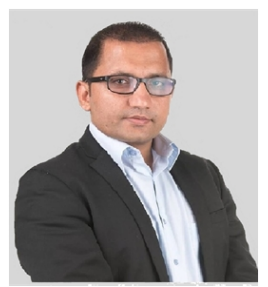

Muddesar Iqbal is Senior Lecturer in Mobile Computing in the Division of Computer Science, School of Engineering. He completed his $\mathrm{PhD}$ (Funded by EPSRC Doctoral Training Award for 4G-based Reconfigurable Mobile Healthcare System) in 2010 from Kingston University UK. He has won more than $15 \mathrm{R} \& \mathrm{D}$ and Capacity building funding Grants from different national and international funding agencies, co-Invented 4 patented inventions in the area of IoT, Intelligent systems and 5G enabling Technologies, published nearly 100 peer-reviewed articles in reputed journals, conference proceedings and book collections. His research interests include 6G, Internet of Sense for Industry 5.0, Collaborative Cognitive Communication Systems Social and Intelligent Autonomous Machines/Robotics.

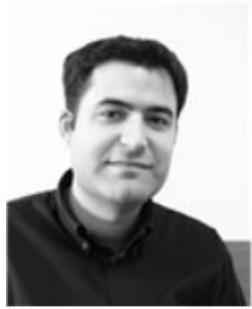

Jonathan Rodriguez Jonathan Rodriguez received his master's degree in Electronic and Electrical Engineering and Ph.D. from the University of Surrey (UK), in 1998 and 2004, respectively. In 2005, he became a Researcher with the Instituto de Telecomunicações (IT), Portugal. In 2017, he was appointed Professor of Mobile Communications at the University of South Wales, UK. He has authored more than 600 scientific works, including 11 book editorials. He is Associate Editor of the IET Communications and IEEE Access journals. He is Chartered Engineer, Fellow of the IET, and Senior Fellow of the Higher Education Academy. 